\title{
GENERAL METHOD OF REGULARIZATION. III: THE UNILATERAL CONTACT PROBLEM
}

Abstract. The aim of this paper is to prove that the relaxation of the elastic-perfectly plastic energy (of a solid made of a Hencky material with the Signorini constraints on the boundary) is the weak* lower semicontinuous regularization of the plastic energy. We consider an elastic-plastic solid endowed with the von Mises (or Tresca) yield condition. Moreover, we show that the set of solutions of the relaxed problem is equal to the set of solutions of the relaxed problem proposed by Suquet. We deduce that the relaxation of the energy of plastic soil (with the Signorini condition on the boundary) is the weak ${ }^{*}$ lower semicontinuous regularization of the energy.

1. Introduction. In this paper we investigate the convex functional

$$
B D \times \mathbb{M}_{b} \ni(\mathbf{u}, \boldsymbol{\mu}) \mapsto \mathcal{S}(\varepsilon(\mathbf{u}), \boldsymbol{\mu})=\int_{\bar{\Omega}} h(x, \varepsilon(\mathbf{u}), \boldsymbol{\mu})
$$

with constraints on the boundary of $\Omega$, where $\varepsilon(\mathbf{u})$ is the symmetrized gradient of $\mathbf{u}, B D(\Omega)$ is the space of bounded deformations and $\boldsymbol{\mu}$ is a regular measure on $\Gamma_{1}, \boldsymbol{\mu} \in \mathbb{M}_{b}\left(\Gamma_{1}, \mathbb{R}^{n}\right)$. Moreover we assume that $\mathcal{S}(\varepsilon(\mathbf{u}), \boldsymbol{\mu})$ $=\infty$ if $\varepsilon(\mathbf{u}) \notin L^{1}$. In [6] we find the lower semicontinuous (l.s.c.) relaxation of $\mathcal{S}$, and we show that the relaxation is a l.s.c. function (in the weak* $B D \times \mathbb{M}_{b}$ topology), not greater than $\mathcal{S}$. Now we prove that the above mentioned relaxation is the largest l.s.c. minorant less than $\mathcal{S}$, in the case of a material with the von Mises (or Tresca) plastic yield condition (i.e. the l.s.c. regularization of $\mathcal{S}$, in this case). Moreover, we consider the Signorini problem in soil mechanics (i.e. a unilateral contact condition on the boundary of a piece of soil).

2000 Mathematics Subject Classification: Primary 49K27; Secondary 49J45, 47N10, 46A20, 26B30, 74C05, 74E05.

Key words and phrases: Hencky plasticity, Signorini problem, soil mechanics, the largest l.s.c. minorant less than the original functional.

Supported by Committee for Scientific Research (Poland) grant No. 2P03A00518. 
The 1.s.c. regularization (in $L_{\text {loc }}^{1}$ topology) of functionals defined on the space $B V(\Omega)$ was investigated in many papers ([1]-[3], [18]), but their authors did not consider problems with constraints on the boundary of $\Omega$.

In [12], the global method of relaxation (cf. [11]) is applied to l.s.c. regularization of quasiconvex functionals with constraints (Dirichlet condition). These functionals are defined on $B V(\Omega)$. The revelant constraints do not describe the relaxation proposed by Suquet (see [22] and [9]) or unilateral contact conditions (cf. condition (H4) from [12]). In [12] the essential assumption of the method is the local coercivity of the density of elastic-plastic energy.

Kohn and Temam [20] solved the existence problem for an elastic-perfectly plastic solid made of a homogeneous Hencky material. To prove that the functional of the total potential energy is weak* l.s.c. in the space $B D(\Omega)$, they used the method of relaxation of the kinematic boundary condition. They did not show that the relaxed problem was the l.s.c. regularization of the original problem.

Unilateral problems in plasticity were considered by Baiocchi, Buttazzo, Gastaldi and Tomarelli [4]. In [4] they solved the Signorini-like Problem, but they were not able to solve the classical Signorini Problem (see [15]) in Hencky plasticity. Tomarelli [25] solved the Signorini problem in the space

$$
U(\Omega) \equiv\left\{\mathbf{u} \in B D(\Omega) \mid \operatorname{div} \mathbf{u} \in L^{2}(\Omega)\right\}, \quad \Omega \subset \mathbb{R}^{3},
$$

for an isotropic and homogeneous body made of a Hencky material.

A 1.s.c. relaxation of the Signorini problem for an anisotropic and nonhomogeneous Hencky material has been found in [6]. Now we prove that the relaxation (of [6]) is the l.s.c. regularization in the case of a material with the von Mises (or Tresca) plastic yield condition. This generalizes Tomarelli's [25] result to the case of a non-homogeneous Hencky material with non-linear elastic law. Moreover, we show that an analogous result holds in soil mechanics (where the set of admissible stresses is a convex cone; see Remark 2).

2. Some basic definitions and theorems. Let $\Omega$ be a bounded, open, connected set of class $C^{1}$ in $\mathbb{R}^{n}$. The space of continuous functions with compact support is denoted by $C_{c}$. Let $C^{\infty}\left(\Omega, \mathbb{R}^{m}\right)$ be the space of $\mathbb{R}^{m}$ valued, infinitely differentiable functions. Moreover, the space of infinitely differentiable functions equal to 0 at the boundary $\operatorname{Fr} \Omega$ of $\Omega$ is denoted by $C_{0}^{\infty}(\Omega)$. Finally, $\mathbb{M}_{b}\left(\Omega, \mathbb{R}^{m}\right)$ is the space of $\mathbb{R}^{m}$-valued, Radon, bounded, regular measures on $\Omega$, with the norm $\|\cdot\|_{\mathbb{M}_{b}\left(\Omega, \mathbb{R}^{m}\right)}$.

We will use the duality pairs $\left(\mathbb{M}_{r}, C_{c}\right)$ or $\left(\mathbb{M}_{b}, C_{0}\right)$, where $\mathbb{M}_{r}$ is the space of regular measures. Duality pairings will be denoted by $\langle\cdot ; \cdot\rangle$, and the scalar product of $\mathbf{z}, \mathbf{z}^{*} \in \mathbb{R}^{n}$ by $\mathbf{z} \cdot \mathbf{z}^{*}$ or $\mathbf{z z}^{*}$. The scalar product of $\mathbf{w}, \mathbf{w}^{*} \in \mathbb{R}^{n \times n}$ is denoted by $\mathbf{w}: \mathbf{w}^{*}=w^{i j} w_{i j}^{*}$. Let $\mathbf{g}=\left(g_{1}, \ldots, g_{m}\right) \in C\left(\bar{\Omega}, \mathbb{R}^{m}\right)$ and 
$\boldsymbol{\mu}=\left(\mu_{1}, \ldots, \mu_{m}\right) \in \mathbb{M}_{b}\left(\Omega, \mathbb{R}^{m}\right)$. Then $\int_{\Omega} \mathbf{g} \cdot \boldsymbol{\mu}=\int_{\Omega} \mathbf{g} \boldsymbol{\mu} \equiv \sum_{i=1}^{m} \int_{\Omega} g_{i} \mu_{i}$. If $F: Y \rightarrow \mathbb{R} \cup\{\infty\}$, then $F^{*}$ denotes its polar function $F^{*}\left(y^{*}\right)=$ $\sup \left\{\left\langle y^{*} ; y\right\rangle-F(y) \mid y \in Y\right\}$, and $\operatorname{dom} F=\{y \in Y \mid F(y)<\infty\}$ (see [16]). If $Q$ is a subset of $Y$, then $I_{Q}(\cdot)$ is its indicator function (taking the value 0 in $Q$ and $\infty$ outside), and $I_{Q}^{*}(\cdot)$ stands for its support function.

Finally, we need the following notations. $B_{V}(\Xi, r)$ is the closed ball in a metric space $V$ with center $\Xi$ and radius $r$. Furthermore, $\operatorname{cl}_{V}(Z)$ stands for the closure of $Z \subset V$ in the topology of the space $V$; analogously, $\mathrm{cl}_{\|\cdot\|}(Z)$ is the closure of the set $Z$ in the norm $\|\cdot\|$. Similarly, $\operatorname{int} Z$ denotes the interior of $Z . \mathcal{L}^{0}\left(\Omega, \mathbb{R}^{m}\right)_{\mu}$ is the set of $\mu$-measurable functions from $\Omega$ into $\mathbb{R}^{m}$. We denote by $\mathbf{E}_{s}^{n}$ the space of symmetric real $n \times n$ matrices. We set $\left\|\left[e_{i j}\right]\right\|_{\mathbf{E}_{s}^{n}} \equiv \sum_{i, j=1}^{n}\left|e_{i j}\right|$. We denote by $\otimes\left(\right.$ resp. $\left.\otimes_{s}\right)$ the tensor product (resp. symmetric tensor product). If $\tau \subset 2^{X}$ is a linear topology in a vector space $X$, then $[X, \tau]$ denotes the topological space and $[X, \tau]^{*}$ its dual. We define the following Banach spaces (see [20], [23]):

$$
\begin{aligned}
& L D(\Omega) \equiv\left\{\mathbf{u} \in L^{1}\left(\Omega, \mathbb{R}^{n}\right) \mid\right. \\
&\left.\varepsilon_{i j}(\mathbf{u}) \equiv \frac{1}{2}\left(\frac{\partial u_{i}}{\partial x_{j}}+\frac{\partial u_{j}}{\partial x_{i}}\right) \in L^{1}(\Omega), i, j=1, \ldots, n\right\}, \\
& B D(\Omega) \equiv\left\{\mathbf{u} \in L^{1}\left(\Omega, \mathbb{R}^{n}\right) \mid \varepsilon_{i j}(\mathbf{u}) \in \mathbb{M}_{b}(\Omega), i, j=1, \ldots, n\right\},
\end{aligned}
$$

with the natural norms

$$
\begin{aligned}
\|\mathbf{u}\|_{L D(\Omega)} & =\|\mathbf{u}\|_{L^{1}}+\sum_{i, j}^{n}\left\|\varepsilon_{i j}(\mathbf{u})\right\|_{L^{1}}, \\
\|\mathbf{u}\|_{B D(\Omega)} & =\|\mathbf{u}\|_{L^{1}}+\sum_{i, j}^{n}\left\|\varepsilon_{i j}(\mathbf{u})\right\|_{\mathbb{M}_{b}} .
\end{aligned}
$$

Moreover, $\mathcal{R}_{0} \equiv\{\mathbf{u} \in B D(\Omega) \mid \boldsymbol{\varepsilon}(\mathbf{u})=\mathbf{0}\}$ denotes the space of rigid motions in $\mathbb{R}^{n}$.

There exists a continuous surjective linear trace $\gamma_{B}:\left[B D(\Omega),\|\cdot\|_{B D}\right] \rightarrow$ $\left[L^{1}\left(\operatorname{Fr} \Omega, \mathbb{R}^{n}\right),\|\cdot\|_{L^{1}}\right]$ such that $\boldsymbol{\gamma}_{B}(\mathbf{u})=\mathbf{u}_{\mid \operatorname{Fr} \Omega}$ for all $\mathbf{u} \in B D(\Omega) \cap C\left(\bar{\Omega}, \mathbb{R}^{n}\right)$ (see $[23])$. We endow the spaces

$$
X \equiv C_{c}\left(\Omega, \mathbb{R}^{n}\right) \times C_{c}\left(\Omega, \mathbf{E}_{s}^{n}\right), \quad X_{0} \equiv\{(\mathbf{g}, \mathbf{h}) \in X \mid \mathbf{g}=\operatorname{div} \mathbf{h}\},
$$

with the natural norm

$$
\begin{aligned}
\|\mathbf{g}\|_{C\left(\Omega, \mathbb{R}^{n}\right)} & +\|\mathbf{h}\|_{C\left(\Omega, \mathbf{E}_{s}^{n}\right)} \\
& \equiv \sup \left\{\|\mathbf{g}(x)\|_{\mathbb{R}^{n}} \mid x \in \Omega\right\}+\sup \left\{\|\mathbf{h}(x)\|_{\mathbf{E}_{s}^{n}} \mid x \in \Omega\right\}
\end{aligned}
$$

for $\mathbf{g} \in C\left(\Omega, \mathbb{R}^{n}\right)$ and $\mathbf{h} \in C\left(\Omega, \mathbf{E}_{s}^{n}\right)$. Then $B D(\Omega)$ is isomorphic to the dual of $\left[\left(X / X_{0}\right),\|\cdot\|_{C\left(\Omega, \mathbb{R}^{n}\right)}+\|\cdot\|_{C\left(\Omega, \mathbf{E}_{s}^{n}\right)}\right]$ (see $\left.[24]\right)$. The topology $\sigma\left(\left(X / X_{0}\right)^{*}, X\right)$ $=\sigma\left(B D(\Omega), C_{c}\left(\Omega, \mathbb{R}^{n}\right) \times C_{c}\left(\Omega, \mathbf{E}_{s}^{n}\right)\right)$ is called the weak $k^{*} B D$ topology. A net 
$\left\{\mathbf{u}_{\delta}\right\}_{\delta \in D} \subset B D(\Omega)$ is convergent to $\mathbf{u}_{0} \in B D(\Omega)$ in this topology if and only if

$$
\int_{\Omega} \mathbf{g} \cdot\left(\mathbf{u}_{0}-\mathbf{u}_{\delta}\right) d x+\int_{\Omega} \mathbf{h}: \boldsymbol{\varepsilon}\left(\mathbf{u}_{0}-\mathbf{u}_{\delta}\right) \rightarrow 0
$$

for all $(\mathbf{g}, \mathbf{h}) \in X$ (see $\left[17\right.$, pp. 73-81]). For every $\varphi \in L^{1}\left(\operatorname{Fr} \Omega, \mathbb{R}^{n}\right)$, the set $\left\{\mathbf{u} \in B D(\Omega) \mid \gamma_{B}(\mathbf{u})=\boldsymbol{\varphi}\right\}$ is dense in $[B D(\Omega)$, weak* topology] (see $[6$, Proposition 2.5]). Then the trace operator $\gamma_{B}$ is not continuous on $[B D(\Omega)$, weak ${ }^{*}$ topology] if the space $L^{1}\left(\operatorname{Fr} \Omega, \mathbb{R}^{n}\right)$ is endowed with a Hausdorff topo$\log y$.

A net $\left\{\mathbf{u}_{\delta}\right\}_{\delta \in D}$ converges to $\mathbf{u}_{0}$ (in the topology (2.7)-(2.8)) if

$$
\begin{aligned}
& \mathbf{u}_{\delta} \rightarrow \mathbf{u}_{0} \quad \text { in }\|\cdot\|_{L^{p}\left(\Omega, \mathbb{R}^{n}\right) \quad \forall p \text { such that } 1 \leq p<q=n /(n-1)} \\
& \quad \text { and weakly in } L^{q}\left(\Omega, \mathbb{R}^{n}\right)(q=\infty \text { if } n=1), \\
& \boldsymbol{\varepsilon}\left(\mathbf{u}_{\delta}\right) \rightarrow \boldsymbol{\varepsilon}\left(\mathbf{u}_{0}\right) \quad \text { weak* in } \mathbb{M}_{b}\left(\Omega, \mathbf{E}_{s}^{n}\right) .
\end{aligned}
$$

The weak* $B D(\Omega)$ topology and the topology (2.7)-(2.8) are equivalent on bounded subsets of $B D(\Omega)$ (cf. Proposition 2 of [8]). Therefore the injection of $\left[B D(\Omega)\right.$, weak $\left.{ }^{*}\right]$ into $\left[L^{p}\left(\Omega, \mathbb{R}^{n}\right)\right.$, weak topology] is continuous on bounded subsets of $B D(\Omega)$, where $1 \leq p \leq q=n /(n-1)(q=\infty$ if $n=1)$.

We define the Banach space of measurable functions

$$
W^{n}(\Omega, \operatorname{div}) \equiv\left\{\boldsymbol{\sigma} \in L^{\infty}\left(\Omega, \mathbf{E}_{s}^{n}\right) \mid \operatorname{div} \boldsymbol{\sigma} \in L^{n}\left(\Omega, \mathbb{R}^{n}\right)\right\}
$$

endowed with the natural norm $\|\boldsymbol{\sigma}\|_{W^{n}(\Omega, \operatorname{div})}=\|\boldsymbol{\sigma}\|_{L^{\infty}\left(\Omega, \mathbf{E}_{s}^{n}\right)}+\|\operatorname{div} \boldsymbol{\sigma}\|_{L^{n}\left(\Omega, \mathbb{R}^{n}\right)}$ (cf. [23, Chap. 2, Sec. 7] and [6]). The distribution $\boldsymbol{\sigma}: \boldsymbol{\varepsilon}(\mathbf{u})$, where $\boldsymbol{\sigma} \in$ $W^{n}(\Omega, \operatorname{div}), \mathbf{u} \in B D(\Omega)$, defined (for $\varphi_{1} \in C_{0}^{\infty}(\Omega)$ ) by

$$
\left\langle\boldsymbol{\sigma}: \boldsymbol{\varepsilon}(\mathbf{u}) ; \varphi_{1}\right\rangle_{D^{\prime} \times D}=-\int_{\Omega}(\operatorname{div} \boldsymbol{\sigma}) \cdot \mathbf{u} \varphi_{1} d x-\int_{\Omega} \boldsymbol{\sigma}:\left(\mathbf{u} \otimes \nabla \varphi_{1}\right) d x,
$$

is a bounded measure on $\Omega$, absolutely continuous with respect to $|\varepsilon(\mathbf{u})|$ (see $[23])$.

Assumption 1. $\Omega$ and $\Omega_{1}$ are bounded open connected sets of class $C^{1}$ in $\mathbb{R}^{n}$ with $\Omega \subset \subset \Omega_{1}$.

THEOREM 1 (cf. [23]). There exists a continuous, linear, surjective, open map $\boldsymbol{\beta}_{B}$ from $\left[W^{n}(\Omega, \operatorname{div}),\|\cdot\|_{W^{n}(\Omega, \operatorname{div})}\right]$ onto $\left[L^{\infty}\left(\operatorname{Fr} \Omega, \mathbb{R}^{n}\right),\|\cdot\|_{L^{\infty}}\right]$ such that $\boldsymbol{\beta}_{B}(\boldsymbol{\sigma})=\boldsymbol{\sigma}_{\mid \operatorname{Fr} \Omega} \cdot \boldsymbol{\nu}$ for every $\boldsymbol{\sigma} \in C\left(\bar{\Omega}, \mathbf{E}_{s}^{n}\right)$, where $\boldsymbol{\nu}$ denotes the exterior unit vector normal to $\operatorname{Fr} \Omega$. Furthermore, for all $\mathbf{u} \in B D(\Omega)$ and all $\boldsymbol{\sigma} \in$ $W^{n}(\Omega$, div), the following Green formula holds:

$$
\int_{\Omega} \boldsymbol{\sigma}: \boldsymbol{\varepsilon}(\mathbf{u})+\int_{\Omega}(\operatorname{div} \boldsymbol{\sigma}) \cdot \mathbf{u} d x=\int_{\operatorname{Fr} \Omega} \boldsymbol{\beta}_{B}(\boldsymbol{\sigma}) \cdot \boldsymbol{\gamma}_{B}(\mathbf{u}) d s .
$$

3. Auxiliary theorems and spaces. In this paper, the Lebesgue and Hausdorff measures on $\Omega$ and $\operatorname{Fr} \Omega$ are denoted by $d x$ and $d s$, respectively. 
Assumption 2 (cf. [8] and [6]). $\mathcal{K}: \bar{\Omega} \rightarrow 2^{\mathbf{E}_{s}^{n}}$ is a multifunction such that $\mathcal{K}(x)$ is a convex closed subset of $\mathbf{E}_{s}^{n}$ for all $x \in \bar{\Omega}$, there exists $\mathbf{z}_{0} \in$ $C^{1}\left(\bar{\Omega}, \mathbf{E}_{s}^{n}\right)$ such that $\mathbf{z}_{0}(x) \in \mathcal{K}(x)$ for every $x \in \bar{\Omega}$, and the following conditions hold:

(i) if $\mathbf{z}(x) \in \mathcal{K}(x)$ for $d x$-almost every (dx-a.e.) $x \in \Omega, \mathbf{z} \in C\left(\bar{\Omega}, \mathbf{E}_{s}^{n}\right)$ and $\mathbf{z}_{\mid \text {int } \Omega} \in W^{n}(\Omega, \operatorname{div})$, then $\mathbf{z}(y) \in \mathcal{K}(y)$ for every $y \in \bar{\Omega}$;

(ii) for every $y \in \bar{\Omega}$ and every $\mathbf{w} \in \mathcal{K}(y)$ there exists $\mathbf{z} \in C\left(\bar{\Omega}, \mathbf{E}_{s}^{n}\right)$ such that $\mathbf{z}_{\mid \text {int } \Omega} \in W^{n}(\Omega, \operatorname{div}), \mathbf{z}(y)=\mathbf{w}$ and $\mathbf{z}(x) \in \mathcal{K}(x)$ for every $x \in \bar{\Omega}$.

Conditions (i) and (ii) are equivalent to the condition that for every $y \in \bar{\Omega}$,

$$
\begin{aligned}
\mathcal{K}(y)=\left\{\mathbf{z}(y) \mid \mathbf{z} \in C\left(\bar{\Omega}, \mathbf{E}_{s}^{n}\right),\right. & \mathbf{z}_{\mid \text {int } \Omega} \in W^{n}(\Omega, \text { div }), \\
& \mathbf{z}(x) \in \mathcal{K}(x) \text { for } d x \text {-a.e. } x \in \Omega\} .
\end{aligned}
$$

Definition 1. Let $j^{*}: \Omega \times \mathbf{E}_{s}^{n} \rightarrow \mathbb{R} \cup\{\infty\}$ be a convex normal integrand (cf. [16, Chapter 8, p. 232] and [8, Definition 2]) such that

$$
\left\{\mathbf{w}^{*} \in \mathbf{E}_{s}^{n} \mid j^{*}\left(x, \mathbf{w}^{*}\right)<\infty\right\}=\mathcal{K}(x) \quad \text { for } d x \text {-a.e. } x \in \Omega .
$$

Assumpion 3. For every $\widehat{r}>0$ there exists $c_{\widehat{r}}$ such that

$$
\begin{aligned}
& \sup \left\{\int_{\Omega} j^{*}\left(x, \mathbf{z}^{*}\right) d x \mid \mathbf{z}^{*} \in L^{\infty}\left(\Omega, \mathbf{E}_{s}^{n}\right),\left\|\mathbf{z}^{*}\right\|_{L^{\infty}}<\widehat{r}\right. \\
& \text { and } \left.\mathbf{z}^{*}(x) \in \mathcal{K}(x) \text { for } d x \text {-a.e. } x \in \Omega\right\}<c_{\widehat{r}}<\infty .
\end{aligned}
$$

Assumption 4. There exist $\mathbf{u}^{e} \in L D(\Omega)$ and $q \in L^{1}(\Omega, \mathbb{R})$ such that $j^{*}\left(x, \mathbf{w}^{*}\right) \geq \varepsilon\left(\mathbf{u}^{e}\right)(x): \mathbf{w}^{*}+q(x)$ for $d x$-a.e. $x \in \Omega$, for every $\mathbf{w}^{*} \in \mathbf{E}_{s}^{n}$ and $\gamma_{B}\left(\mathbf{u}^{e}\right)=\mathbf{0}$ on $\operatorname{Fr} \Omega$.

The set $\mathcal{K}(x)$ denotes the elasticity convex domain at the point $x$. Define

$$
j(x, \mathbf{w}) \equiv j^{* *}(x, \mathbf{w}) \equiv \sup \left\{\mathbf{w}: \mathbf{w}^{*}-j^{*}\left(x, \mathbf{w}^{*}\right) \mid \mathbf{w}^{*} \in \mathbf{E}_{s}^{n}\right\}
$$

for $d x$-a.e. $x \in \Omega$ and all $\mathbf{w} \in \mathbf{E}_{s}^{n}$. Then $j$ is a convex normal integrand. Define $j_{\infty}: \bar{\Omega} \times \mathbf{E}_{s}^{n} \rightarrow \mathbb{R} \cup\{\infty\}$ by

$$
j_{\infty}(x, \mathbf{w}) \equiv \sup \left\{\mathbf{w}: \mathbf{w}^{*}-I_{\mathcal{K}(x)}\left(\mathbf{w}^{*}\right) \mid \mathbf{w}^{*} \in \mathbf{E}_{s}^{n}\right\}
$$

for $x \in \bar{\Omega}$ and $\mathbf{w} \in \mathbf{E}_{s}^{n}$.

Assumption 5. $\Gamma_{0}, \Gamma_{1}$ and $\Gamma_{s}$ are Borel subsets of Fr $\Omega$ such that $\Gamma_{0} \cap$ $\Gamma_{1}=\emptyset, \Gamma_{0} \cap \Gamma_{s}=\emptyset, \Gamma_{s} \cap \Gamma_{1}=\emptyset, \Gamma_{1}=\bar{\Gamma}_{1}$,ds(int $\left.\Gamma_{s}\right)=d s\left(\bar{\Gamma}_{s}\right)$ and $d s\left(\operatorname{Fr} \Omega-\left(\Gamma_{0} \cup \Gamma_{1} \cup \Gamma_{s}\right)\right)=0$. Moreover, $\Gamma_{1}=\operatorname{Fr} \Omega \cap \mathcal{C}$, where $\mathcal{C}=\operatorname{clint} \mathcal{C} \subset \Omega_{1}$ is a closed Caccioppoli set and $d s(\operatorname{Fr} \Omega \cap \operatorname{Fr} \mathcal{C})=0$ (see [19], [8, Definition 3]). Furthermore, $\gamma_{B}(\mathbf{u})_{\mid \Gamma_{0}}=\mathbf{0}$ on $\Gamma_{0}$ and an $(n-1)$-dimensional density force $\mathbf{g} \in L^{\infty}\left(\Gamma_{1}, \mathbb{R}^{n}\right)$ is prescribed on $\Gamma_{1}$. Moreover, $\boldsymbol{\gamma}_{B}(\mathbf{u})(x) \cdot \boldsymbol{\nu}(x) \leq 0$ for $d s$-a.e. $x \in \Gamma_{s}$. This means that we have a potential function $h_{s}: \Gamma_{s} \times \mathbf{E}_{s}^{n} \rightarrow \mathbb{R} \cup\{\infty\}$, defined by 


$$
h_{s}(x, \mathbf{y})= \begin{cases}\infty & \text { if } \sum_{i=1}^{n} y_{i i}<0 \\ 0 & \text { otherwise }\end{cases}
$$

where $\mathbf{y}=-\gamma_{B}(\mathbf{u}) \otimes_{s} \boldsymbol{\nu}$.

The displacement formulation of the equilibrium problem for the elasticperfectly plastic body made of a Hencky material with the Signorini contact condition, reads (see [6]):

$$
\left(S N_{\lambda, j}\right) \quad \text { Find } \inf \left\{\lambda F_{1}(\mathbf{u})+G_{j}(\varepsilon(\mathbf{u})) \mid \mathbf{u} \in L D(\Omega)\right\},
$$

where

$$
\begin{aligned}
& \lambda F_{1}(\mathbf{u}) \equiv-\lambda\left(\int_{\Omega} \mathbf{f} \cdot \mathbf{u} d x+\int_{\Gamma_{1}} \mathbf{g} \cdot \boldsymbol{\gamma}_{B}(\mathbf{u}) d s\right) \\
& \quad+\int_{\Gamma_{0}} I_{\left\{\boldsymbol{\gamma}_{B}(\mathbf{u})(x)=\mathbf{0}\right\}}\left(-\boldsymbol{\gamma}_{B}(\mathbf{u}) \otimes_{s} \boldsymbol{\nu}\right) d s+\int_{\Gamma_{s}} h_{s}\left(x,-\boldsymbol{\gamma}_{B}(\mathbf{u}) \otimes_{s} \boldsymbol{\nu}\right) d s
\end{aligned}
$$

for $\mathbf{u} \in B D(\Omega)$, and the functional $G_{j}(\varepsilon(\cdot)): B D(\Omega) \rightarrow \mathbb{R} \cup\{\infty\}$ is given by

$$
G_{j}(\varepsilon(\mathbf{u})) \equiv \begin{cases}\int_{\Omega} j(x, \boldsymbol{\varepsilon}(\mathbf{u})) d x & \text { if } \mathbf{u} \in L D(\Omega), \\ \infty & \text { if } \mathbf{u} \in B D(\Omega)-L D(\Omega) .\end{cases}
$$

The quantity in (3.7) describes the total elastic-perfectly plastic energy of a body occupying the given subset $\Omega$ of $\mathbb{R}^{n}$. This body is subjected to volume forces $\mathbf{f} \in L^{n}\left(\Omega, \mathbb{R}^{n}\right)$ and boundary forces $\mathbf{g} \in L^{\infty}\left(\Gamma_{1}, \mathbb{R}^{n}\right)$. The constant $\lambda \geq 0, \lambda<\infty$, is the load multiplier (see [23, Chap. 1, Sec. 4]). The body is clamped on $\Gamma_{0}$. We assume the Signorini contact condition on $\Gamma_{s}$.

Similarly, we define the Suquet unilateral contact problem (cf. [22]):

$$
\left(S S_{\lambda, j}\right) \quad \text { Find } \inf \left\{\lambda F_{2}(\mathbf{u}, \boldsymbol{\mu})+G_{j}(\varepsilon(\mathbf{u})) \mid\right.
$$

$$
\left.(\mathbf{u}, \boldsymbol{\mu}) \in L D(\Omega) \times \mathbb{M}_{b}\left(\Gamma_{1}, \mathbb{R}^{n}\right)\right\},
$$

where

$$
\begin{aligned}
& \lambda F_{2}(\mathbf{u}, \boldsymbol{\mu}) \equiv-\lambda\left(\int_{\Omega} \mathbf{f} \cdot \mathbf{u} d x+\int_{\Gamma_{1}} \mathbf{g} \cdot \boldsymbol{\mu}\right) \\
& +\int_{\Gamma_{0}} I_{\left\{\boldsymbol{\gamma}_{B}(\mathbf{u})(x)=\mathbf{0}\right\}}\left(-\boldsymbol{\gamma}_{B}(\mathbf{u}) \otimes_{s} \boldsymbol{\nu}\right) d s+\int_{\Gamma_{s}} h_{s}\left(x,-\boldsymbol{\gamma}_{B}(\mathbf{u}) \otimes_{s} \boldsymbol{\nu}\right) d s
\end{aligned}
$$

if $\mathbf{u} \in L D(\Omega), \boldsymbol{\mu}=\boldsymbol{\gamma}_{B}(\mathbf{u}) d s$ for $\left|\boldsymbol{\mu}-\boldsymbol{\gamma}_{B}(\mathbf{u}) d s\right|$-a.e. $x \in \Gamma_{1}$, and $\lambda F_{2}(\mathbf{u}, \boldsymbol{\mu})$ $\equiv \infty$ otherwise. Unfortunately, $\left(S N_{\lambda, j}\right)$ and $\left(S S_{\lambda, j}\right)$ are not l.s.c. in the weak $^{*} B D(\Omega)$ and weak ${ }^{*} B D(\Omega) \times \mathbb{M}_{b}\left(\Gamma_{1}, \mathbb{R}^{n}\right)$ topologies, respectively (cf. $[9])$. Below we obtain explicitly the relaxed form of $\left(S S_{\lambda, j}\right)$ and show the relaxed form of $\left(S N_{\lambda, j}\right)$.

Let $\boldsymbol{\mu} \in \mathbb{M}_{b}\left(\Omega, \mathbf{E}_{s}^{n}\right)$. We recall that $|\boldsymbol{\mu}|$ is the total variation measure associated with $\boldsymbol{\mu}$. The density of $\boldsymbol{\mu}$ with respect to $|\boldsymbol{\mu}|$ will be denoted by $d \boldsymbol{\mu} / d|\boldsymbol{\mu}|$. Let $\boldsymbol{\mu}=\boldsymbol{\mu}_{a}(x) d x+\boldsymbol{\mu}_{s}$ be the Lebesgue decomposition of $\boldsymbol{\mu}$ into the absolutely continuous and singular parts with respect to $d x$. 
We consider the spaces $\mathbf{Y}^{1}(\bar{\Omega})$ and $C_{\operatorname{div}}\left(\bar{\Omega}, \mathbf{E}_{s}^{n}\right)$, given by

$$
\begin{gathered}
\mathbf{Y}^{1}(\bar{\Omega}) \equiv\left\{\mathbf{M} \in \mathbb{M}_{b}\left(\bar{\Omega}, \mathbf{E}_{s}^{n}\right) \mid \exists \mathbf{u}_{1} \in B D\left(\Omega_{1}\right),\right. \\
\left.\varepsilon\left(\mathbf{u}_{1}\right)_{\mid \bar{\Omega}}=\mathbf{M}, \mathbf{u}_{1 \mid \Omega_{1}-\bar{\Omega}}=\mathbf{0}\right\}, \\
C_{\operatorname{div}}\left(\bar{\Omega}, \mathbf{E}_{s}^{n}\right) \equiv\left\{\boldsymbol{\sigma} \in C\left(\bar{\Omega}, \mathbf{E}_{s}^{n}\right) \mid \boldsymbol{\sigma}_{\mid \Omega} \in W^{n}(\Omega, \operatorname{div})\right\} .
\end{gathered}
$$

These are topological vector spaces placed in duality by the bilinear pairing

$$
\langle\mathbf{M} ; \boldsymbol{\sigma}\rangle_{\mathbf{Y}^{1} \times C\left(\bar{\Omega}, \mathbf{E}_{s}^{n}\right)}=\int_{\bar{\Omega}} \boldsymbol{\sigma}: \mathbf{M}=\sum_{i, j=1}^{n} \int_{\bar{\Omega}} \sigma_{i j} M^{i j} .
$$

We say that a sequence $\left\{\mathbf{M}_{m}\right\}_{m \in \mathbb{N}} \subset \mathbf{Y}^{1}(\bar{\Omega})$ converges to $\mathbf{M}_{0}$ in the topology $\sigma\left(\mathbf{Y}^{1}(\bar{\Omega}), C_{\operatorname{div}}\left(\bar{\Omega}, \mathbf{E}_{s}^{n}\right)\right)$ if $\left\langle\left(\mathbf{M}_{m}-\mathbf{M}_{0}\right) ; \boldsymbol{\sigma}\right\rangle_{\mathbf{Y}^{1} \times C\left(\bar{\Omega}, \mathbf{E}_{s}^{n}\right)} \rightarrow 0$ for every $\boldsymbol{\sigma} \in$ $C_{\text {div }}\left(\bar{\Omega}, \mathbf{E}_{s}^{n}\right)$ as $m \rightarrow \infty$. By [14, Theorem V.3.9] we have [ $\mathbf{Y}^{1}(\bar{\Omega}), \sigma\left(\mathbf{Y}^{1}(\bar{\Omega})\right.$, $\left.\left.C_{\operatorname{div}}\left(\bar{\Omega}, \mathbf{E}_{s}^{n}\right)\right)\right]^{*}=C_{\operatorname{div}}\left(\bar{\Omega}, \mathbf{E}_{s}^{n}\right)$.

Assumption 6. There exists $\boldsymbol{\sigma}_{0} \in C_{\text {div }}\left(\bar{\Omega}, \mathbf{E}_{s}^{n}\right)$ for which $\boldsymbol{\beta}_{B}\left(\boldsymbol{\sigma}_{0}\right)=\lambda \mathbf{g}$ on $\Gamma_{1}$ and $\boldsymbol{\sigma}_{0}(x) \in \mathcal{K}(x)$ for $d x$-a.e. $x \in \Omega$.

The space $B D(\Omega)$ is isomorphic to $\mathcal{A}_{B D} \equiv\left\{\mathbf{u} \in B D\left(\Omega_{1}\right) \mid \mathbf{u}_{\mid \Omega_{1}-\bar{\Omega}}=\mathbf{0}\right\}$ (cf. Assumption 1). Moreover, $\mathcal{A}_{B D}$ is isomorphic to $\mathbf{Y}^{1}(\bar{\Omega})$, and the isomorphism is given by $\mathcal{A}_{B D} \ni \mathbf{u} \mapsto \varepsilon(\mathbf{u})_{\mid \bar{\Omega}} \in \mathbf{Y}^{1}(\bar{\Omega})$. The Banach spaces $\left[B D(\Omega),\|\cdot\|_{B D}\right]$ and $\left[\mathbf{Y}^{1}(\bar{\Omega}),\|\cdot\|_{\mathbb{M}_{b}(\bar{\Omega})}\right]$ are isomorphic (cf. [6, Proposition 4.24]). Each closed ball $\mathrm{cl}_{\|\cdot\|}\left(B(0, r)\right.$ ) (in $\mathbf{Y}^{1}$ ) is compact in the topology $\sigma\left(\mathbf{Y}^{1}(\bar{\Omega}), C_{\operatorname{div}}\left(\bar{\Omega}, \mathbf{E}_{s}^{n}\right)\right)$, where $\mathrm{cl}_{\|\cdot\|}$ denotes the closure in the norm of $B D(\Omega)$ (see $[6$, Proposition 4.23$])$. The space $\left[\operatorname{cl}_{\|\cdot\|_{B D}}\left(B_{B D}(0, r)\right)\right.$, weak $^{*} B D(\Omega)$ topology $]$ is isomorphic to $\left[\operatorname{cl}_{\|\cdot\|_{B D}}\left(B_{B D}(0, r)\right), \sigma\left(\mathbf{Y}^{1}(\bar{\Omega})\right.\right.$, $\left.\left.C_{\text {div }}\left(\bar{\Omega}, \mathbf{E}_{s}^{n}\right)\right)\right]$ (cf. [6, Proposition 4.25]). Let

$$
\mathbf{M}^{1}\left(\Gamma_{1}\right) \equiv\left\{\boldsymbol{\mu} \otimes_{s} \boldsymbol{\nu} \in \mathbb{M}_{b}\left(\Gamma_{1}, \mathbf{E}_{s}^{n}\right) \mid \boldsymbol{\mu} \in \mathbb{M}_{b}\left(\Gamma_{1}, \mathbb{R}^{n}\right)\right\} .
$$

Consider the vector spaces

$$
\begin{aligned}
& {\left[C\left(\Gamma_{1}, \mathbf{E}_{s}^{n}\right), \sigma\left(C\left(\Gamma_{1}, \mathbf{E}_{s}^{n}\right), \mathbf{M}^{1}\left(\Gamma_{1}\right)\right)\right],} \\
& {\left[\mathbf{M}^{1}\left(\Gamma_{1}\right), \sigma\left(\mathbf{M}^{1}\left(\Gamma_{1}\right), C\left(\Gamma_{1}, \mathbf{E}_{s}^{n}\right)\right)\right]}
\end{aligned}
$$

(the latter being equivalent to $\left[\mathbf{M}^{1}\left(\Gamma_{1}\right)\right.$, $\left.\left.\operatorname{weak}^{*} \mathbf{M}^{1}\left(\Gamma_{1}\right)\right]\right)$.

The inside (resp. outside) trace of $B D(\Omega)$ into $L^{1}\left(\operatorname{Fr} \Omega, \mathbb{R}^{n}\right.$ ) (resp. $B D\left(\Omega_{1}-\bar{\Omega}\right)$ into $L^{1}\left(\operatorname{Fr} \Omega, \mathbb{R}^{n}\right)$ ) is denoted by $\gamma_{B}^{I}\left(\right.$ resp. $\gamma_{B}^{O}$ ) (see [23]).

The functional $\mathcal{S}_{\lambda}^{j}: \mathbf{Y}^{1}(\bar{\Omega}) \times \mathbf{M}^{1}\left(\Gamma_{1}\right) \rightarrow \mathbb{R} \cup\{\infty\}$ is defined by

$$
\begin{aligned}
& \mathcal{S}_{\lambda}^{j}\left(\varepsilon(\mathbf{u})_{\mid \bar{\Omega}}, \boldsymbol{\mu} \otimes_{s} \boldsymbol{\nu}\right) \equiv-\int_{\Gamma_{1}} \lambda \mathbf{g} \cdot \boldsymbol{\mu}+\int_{\Omega} j\left(x, \boldsymbol{\varepsilon}(\mathbf{u})_{a}\right) d x \\
& +\int_{\Gamma_{0}} I_{\left\{\left(\boldsymbol{\gamma}_{B}^{I}(\mathbf{u}) \otimes_{s} \boldsymbol{\nu}\right)(\mathbf{x})=\mathbf{0}\right\}}\left(\boldsymbol{\gamma}_{B}^{I}(\mathbf{u}) \otimes_{s} \boldsymbol{\nu}\right) d s+\int_{\Gamma_{s}} h_{s}\left(x,-\gamma_{B}^{I}(\mathbf{u}) \otimes_{s} \boldsymbol{\nu}\right) d s
\end{aligned}
$$


if $\mathbf{u}_{\mid \Omega} \in L D(\Omega)$ and $\boldsymbol{\mu}=\gamma_{B}^{I}(\mathbf{u}) d s$ for $\left|\boldsymbol{\mu}-\gamma_{B}^{I}(\mathbf{u}) d s\right|$-a.e. $x \in \Gamma_{1}$, and $\mathcal{S}_{\lambda}^{j}\left(\varepsilon(\mathbf{u})_{\mid \bar{\Omega}}, \boldsymbol{\mu} \otimes_{s} \boldsymbol{\nu}\right) \equiv \infty$ otherwise, where $\boldsymbol{\varepsilon}(\mathbf{u})_{\mid \bar{\Omega}} \in \mathbf{Y}^{1}(\bar{\Omega})$ and $\boldsymbol{\mu} \in \mathbb{M}_{b}\left(\Gamma_{1}, \mathbb{R}^{n}\right)$ (cf. [6]). We assume that there exist $\widetilde{\mathbf{u}} \in B D\left(\Omega_{1}\right)$ and $\widetilde{\boldsymbol{\mu}} \in \mathbb{M}_{b}\left(\Gamma_{1}, \mathbb{R}^{n}\right)$ such that $\widetilde{\mathbf{u}}_{\mid \Omega} \in L D(\Omega)$ and $\mathcal{S}_{\lambda}^{j}\left(\varepsilon(\widetilde{\mathbf{u}})_{\mid \bar{\Omega}}, \widetilde{\boldsymbol{\mu}} \otimes_{s} \boldsymbol{\nu}\right)<\infty$.

4. Lower semicontinuous regularization. In this section the l.s.c. regularization of the functional $\mathcal{S}_{\lambda}^{j}$ is found, where the spaces $B D(\Omega)$ and $\mathbf{M}^{1}\left(\Gamma_{1}\right)$ are endowed with the weak ${ }^{*} B D$ topology and $\sigma\left(\mathbf{M}^{1}\left(\Gamma_{1}\right), C\left(\Gamma_{1}, \mathbf{E}_{s}^{n}\right)\right)$ topology, respectively. We apply this result to the problem of relaxation in Hencky plasticity.

The bilinear form between $\mathbf{Y}^{1}(\bar{\Omega}) \times \mathbf{M}^{1}\left(\Gamma_{1}\right)$ and $C_{\mathrm{div}}\left(\bar{\Omega}, \mathbf{E}_{s}^{n}\right) \times C\left(\Gamma_{1}, \mathbf{E}_{s}^{n}\right)$ is given by

$$
\begin{aligned}
\left\langle\left(\varepsilon(\mathbf{u})_{\mid \bar{\Omega}}, \boldsymbol{\mu} \otimes_{s} \boldsymbol{\nu}\right) ;(\boldsymbol{\sigma}, \boldsymbol{\kappa})\right\rangle_{2} & \equiv \int_{\Omega} \boldsymbol{\sigma}: \boldsymbol{\varepsilon}(\mathbf{u})_{\mid \Omega} \\
& +\int_{\operatorname{Fr} \Omega} \boldsymbol{\sigma}:\left(-\gamma_{B}^{I}(\mathbf{u}) \otimes_{s} \boldsymbol{\nu}\right) d s+\int_{\Gamma_{1}} \boldsymbol{\kappa}:\left[\boldsymbol{\mu} \otimes_{s} \boldsymbol{\nu}\right]
\end{aligned}
$$

for $\varepsilon(\mathbf{u})_{\mid \bar{\Omega}}=\left(\varepsilon(\mathbf{u})_{\mid \Omega},\left(-\gamma_{B}^{I}(\mathbf{u}) d s \otimes_{s} \boldsymbol{\nu}\right)\right) \in \mathbf{Y}^{1}(\bar{\Omega}), \boldsymbol{\mu} \otimes_{s} \boldsymbol{\nu} \in \mathbf{M}^{1}\left(\Gamma_{1}\right), \boldsymbol{\sigma} \in$ $C_{\mathrm{div}}\left(\bar{\Omega}, \mathbf{E}_{s}^{n}\right)$ and $\boldsymbol{\kappa} \in C\left(\Gamma_{1}, \mathbf{E}_{s}^{n}\right)$ (cf. Proposition 6 of [7]). Because of the duality between $\mathbf{Y}^{1}(\bar{\Omega}) \times \mathbf{M}^{1}\left(\Gamma_{1}\right)$ and $C_{\mathrm{div}}\left(\bar{\Omega}, \mathbf{E}_{s}^{n}\right) \times C\left(\Gamma_{1}, \mathbf{E}_{s}^{n}\right)$ we define a functional $\left(\mathcal{S}_{\lambda}^{j}\right)^{*}: C_{\operatorname{div}}\left(\bar{\Omega}, \mathbf{E}_{s}^{n}\right) \times C\left(\Gamma_{1}, \mathbf{E}_{s}^{n}\right) \rightarrow \mathbb{R} \cup\{\infty\}$ by

$$
\begin{aligned}
& \left(\mathcal{S}_{\lambda}^{j}\right)^{*}(\boldsymbol{\sigma}, \boldsymbol{\kappa}) \equiv \sup \left\{\left\langle\left(\varepsilon(\mathbf{u})_{\mid \bar{\Omega}}, \boldsymbol{\mu} \otimes_{s} \boldsymbol{\nu}\right) ;(\boldsymbol{\sigma}, \boldsymbol{\kappa})\right\rangle_{2}\right. \\
& \left.\quad-\mathcal{S}_{\lambda}^{j}\left(\varepsilon(\mathbf{u})_{\mid \bar{\Omega}}, \boldsymbol{\mu} \otimes_{s} \boldsymbol{\nu}\right) \mid \boldsymbol{\varepsilon}(\mathbf{u})_{\mid \bar{\Omega}} \in \mathbf{Y}^{1}(\bar{\Omega}), \boldsymbol{\mu} \otimes_{s} \boldsymbol{\nu} \in \mathbf{M}^{1}\left(\Gamma_{1}\right)\right\}
\end{aligned}
$$

for $\boldsymbol{\sigma} \in C_{\operatorname{div}}\left(\bar{\Omega}, \mathbf{E}_{s}^{n}\right)$ and $\boldsymbol{\kappa} \in C\left(\Gamma_{1}, \mathbf{E}_{s}^{n}\right)$. The bidual functional $\left(\mathcal{S}_{\lambda}^{j}\right)^{* *}$ : $\mathbf{Y}^{1}(\bar{\Omega}) \times \mathbf{M}^{1}\left(\Gamma_{1}\right) \rightarrow \mathbb{R} \cup\{\infty\}$ is defined by

$(4.3)\left(\mathcal{S}_{\lambda}^{j}\right)^{* *}\left(\varepsilon(\mathbf{u})_{\mid \bar{\Omega}}, \boldsymbol{\mu} \otimes_{s} \boldsymbol{\nu}\right)=\sup \left\{\left\langle\left(\varepsilon(\mathbf{u})_{\mid \bar{\Omega}}, \boldsymbol{\mu} \otimes_{s} \boldsymbol{\nu}\right) ;(\boldsymbol{\sigma}, \boldsymbol{\kappa})\right\rangle_{2}-\left(\mathcal{S}_{\lambda}^{j}\right)^{*}(\boldsymbol{\sigma}, \boldsymbol{\kappa}) \mid\right.$

$$
\left.\boldsymbol{\sigma} \in C_{\operatorname{div}}\left(\bar{\Omega}, \mathbf{E}_{s}^{n}\right), \boldsymbol{\kappa} \in C\left(\Gamma_{1}, \mathbf{E}_{s}^{n}\right)\right\}
$$

for $\varepsilon(\mathbf{u})_{\mid \bar{\Omega}} \in \mathbf{Y}^{1}(\bar{\Omega})$ and $\boldsymbol{\mu} \in \mathbb{M}_{b}\left(\Gamma_{1}, \mathbb{R}^{n}\right)$.

The bilinear form between $\mathbb{M}_{b}\left(\Omega, \mathbf{E}_{s}^{n}\right) \times \mathbf{Y}^{1}(\bar{\Omega})_{\mid \operatorname{Fr} \Omega} \times \mathbf{M}^{1}\left(\Gamma_{1}\right)$ and $C_{\text {div }}\left(\bar{\Omega}, \mathbf{E}_{s}^{n}\right) \times C\left(\Gamma_{1}, \mathbf{E}_{s}^{n}\right)$ is

$$
\begin{aligned}
\left\langle\left(\mathbf{w},-\gamma_{B}^{I}(\mathbf{u}) \otimes_{s} \boldsymbol{\nu}, \boldsymbol{\mu} \otimes_{s} \boldsymbol{\nu}\right) ;(\boldsymbol{\sigma}, \boldsymbol{\kappa})\right\rangle_{3} \equiv \int_{\Omega} \boldsymbol{\sigma}: \mathbf{w} \\
\quad+\int_{\operatorname{Fr} \Omega} \boldsymbol{\sigma}:\left(-\gamma_{B}^{I}(\mathbf{u}) \otimes_{s} \boldsymbol{\nu}\right) d s+\int_{\Gamma_{1}} \boldsymbol{\kappa}:\left[\mathbf{u} \otimes_{s} \boldsymbol{\nu}\right]
\end{aligned}
$$

for $\mathbf{w} \in \mathbb{M}_{b}\left(\Omega, \mathbf{E}_{s}^{n}\right), \gamma_{B}^{I}(\mathbf{u}) d s \otimes_{s} \boldsymbol{\nu} \in \mathbf{Y}^{1}(\bar{\Omega})_{\mid \operatorname{Fr} \Omega}, \boldsymbol{\mu} \otimes_{s} \boldsymbol{\nu} \in \mathbf{M}^{1}\left(\Gamma_{1}\right), \boldsymbol{\sigma} \in$ $C_{\text {div }}\left(\bar{\Omega}, \mathbf{E}_{s}^{n}\right)$ and $\kappa \in C\left(\Gamma_{1}, \mathbf{E}_{s}^{n}\right)$. The extension of $\mathcal{S}_{\lambda}^{j}$ onto $\mathbb{M}_{b}\left(\Omega, \mathbf{E}_{s}^{n}\right) \times$ $\mathbf{Y}^{1}(\bar{\Omega})_{\mid \operatorname{Fr} \Omega} \times \mathbf{M}^{1}\left(\Gamma_{1}\right)$ (denoted by $\widetilde{\mathcal{S}}_{\lambda}^{j}$ ) is given by 


$$
\begin{aligned}
\widetilde{\mathcal{S}}_{\lambda}^{j}(\mathbf{w}, & \left.-\gamma_{B}^{I}(\mathbf{u}) d s \otimes_{s} \boldsymbol{\nu}, \boldsymbol{\mu} \otimes_{s} \boldsymbol{\nu}\right) \equiv-\int_{\Gamma_{1}} \lambda \mathbf{g} \cdot \boldsymbol{\mu}+\int_{\Omega} j(x, \mathbf{w}) d x \\
& +\int_{\Gamma_{0}} I_{\left\{\boldsymbol{\gamma}_{B}^{I}(\mathbf{u}) \otimes_{s} \boldsymbol{\nu}=\mathbf{0}\right\}}\left(\boldsymbol{\gamma}_{B}^{I}(\mathbf{u}) \otimes_{s} \boldsymbol{\nu}\right) d s+\int_{\Gamma_{s}} h_{s}\left(x,-\boldsymbol{\gamma}_{B}^{I}(\mathbf{u}) \otimes_{s} \boldsymbol{\nu}\right) d s
\end{aligned}
$$

if $\mathbf{w} \in L^{1}\left(\Omega, \mathbf{E}_{s}^{n}\right)$ and $\boldsymbol{\mu}=\boldsymbol{\gamma}_{B}^{I}(\mathbf{u}) d s$ for $\left|\boldsymbol{\mu}-\boldsymbol{\gamma}_{B}^{I}(\mathbf{u}) d s\right|$-a.e. $x \in \Gamma_{1}$, and $\widetilde{\mathcal{S}}_{\lambda}^{j}\left(\mathbf{w},-\gamma_{B}^{I}(\mathbf{u}) d s \otimes_{s} \boldsymbol{\nu}, \boldsymbol{\mu} \otimes_{s} \boldsymbol{\nu}\right) \equiv \infty$ otherwise.

By duality between $\mathbb{M}_{b}\left(\Omega, \mathbf{E}_{s}^{n}\right) \times \mathbf{Y}^{1}(\bar{\Omega})_{\mid \operatorname{Fr} \Omega} \times \mathbf{M}^{1}\left(\Gamma_{1}\right)$ and $C_{\text {div }}\left(\bar{\Omega}, \mathbf{E}_{s}^{n}\right)$ $\times C\left(\Gamma_{1}, \mathbf{E}_{s}^{n}\right)$, we define the functional $\left(\widetilde{\mathcal{S}}_{\lambda}^{j}\right)^{*}: C_{\operatorname{div}}\left(\bar{\Omega}, \mathbf{E}_{s}^{n}\right) \times C\left(\Gamma_{1}, \mathbf{E}_{s}^{n}\right) \rightarrow$ $\mathbb{R} \cup\{\infty\}$. It is given by

$$
\begin{array}{r}
\left(\widetilde{\mathcal{S}}_{\lambda}^{j}\right)^{*}(\boldsymbol{\sigma}, \boldsymbol{\kappa}) \equiv \sup \left\{\left\langle\left(\mathbf{w},-\boldsymbol{\gamma}_{B}^{I}(\mathbf{u}) \otimes_{s} \boldsymbol{\nu}, \boldsymbol{\mu} \otimes_{s} \boldsymbol{\nu}\right) ;(\boldsymbol{\sigma}, \boldsymbol{\kappa})\right\rangle_{3}\right. \\
-\widetilde{\mathcal{S}}_{\lambda}^{j}\left(\mathbf{w},-\gamma_{B}^{I}(\mathbf{u}) d s \otimes_{s} \boldsymbol{\nu}, \boldsymbol{\mu} \otimes_{s} \boldsymbol{\nu}\right) \mid \mathbf{w} \in L^{1}\left(\Omega, \mathbf{E}_{s}^{n}\right), \mathbf{u} \in B D(\Omega) \\
\text { and } \left.\boldsymbol{\mu} \otimes_{s} \boldsymbol{\nu} \in \mathbf{M}^{1}\left(\Gamma_{1}\right)\right\}
\end{array}
$$

for $\boldsymbol{\sigma} \in C_{\operatorname{div}}\left(\bar{\Omega}, \mathbf{E}_{s}^{n}\right)$ and $\boldsymbol{\kappa} \in C\left(\Gamma_{1}, \mathbf{E}_{s}^{n}\right)$. The bidual functional $\left(\widetilde{\mathcal{S}}_{\lambda}^{j}\right)^{* *}$ : $\mathbf{Y}^{1}(\bar{\Omega}) \times \mathbf{M}^{1}\left(\Gamma_{1}\right) \rightarrow \mathbb{R} \cup\{\infty\}$ is defined by

$$
\begin{aligned}
&\left(\widetilde{\mathcal{S}}_{\lambda}^{j}\right)^{* *}\left(\mathbf{w},-\gamma_{B}^{I}(\mathbf{u}) d s \otimes_{s} \boldsymbol{\nu}, \boldsymbol{\mu} \otimes_{s} \boldsymbol{\nu}\right) \\
& \equiv \sup \left\{\left\langle\left(\mathbf{w},-\gamma_{B}^{I}(\mathbf{u}) \otimes_{s} \boldsymbol{\nu}, \boldsymbol{\mu} \otimes_{s} \boldsymbol{\nu}\right) ;(\boldsymbol{\sigma}, \boldsymbol{\kappa})\right\rangle_{3}-\left(\widetilde{\mathcal{S}}_{\lambda}^{j}\right)^{*}(\boldsymbol{\sigma}, \boldsymbol{\kappa}) \mid\right. \\
&\left.\boldsymbol{\sigma} \in C_{\operatorname{div}}\left(\bar{\Omega}, \mathbf{E}_{s}^{n}\right), \boldsymbol{\kappa} \in C\left(\Gamma_{1}, \mathbf{E}_{s}^{n}\right)\right\}
\end{aligned}
$$

for $\left(\mathbf{w},-\gamma_{B}^{I}(\mathbf{u}) d s \otimes_{s} \boldsymbol{\nu}\right) \in \mathbf{Y}^{1}(\bar{\Omega})$ and $\boldsymbol{\mu} \otimes_{s} \boldsymbol{\nu} \in \mathbf{M}^{1}\left(\Gamma_{1}\right)$.

Lemma 2 (see [6] and [8, Lemma 6]). For every $\boldsymbol{\sigma} \in C_{\operatorname{div}}\left(\bar{\Omega}, \mathbf{E}_{s}^{n}\right)$ and $\boldsymbol{\kappa} \in C\left(\Gamma_{1}, \mathbf{E}_{s}^{n}\right)$ we have $\left(\widetilde{\mathcal{S}}_{\lambda}^{j}\right)^{*}(\boldsymbol{\sigma}, \boldsymbol{\kappa}) \geq\left(\mathcal{S}_{\lambda}^{j}\right)^{*}(\boldsymbol{\sigma}, \boldsymbol{\kappa})$. Moreover, $\left(\widetilde{\mathcal{S}}_{\lambda}^{j}\right)^{* *}(\mathbf{M}$, $\left.\boldsymbol{\mu} \otimes_{s} \boldsymbol{\nu}\right) \leq\left(\mathcal{S}_{\lambda}^{j}\right)^{* *}\left(\mathbf{M}, \boldsymbol{\mu} \otimes_{s} \boldsymbol{\nu}\right)$ for every $\mathbf{M} \in \mathbf{Y}^{1}(\bar{\Omega})$ and $\boldsymbol{\mu} \in \mathbb{M}_{b}\left(\Gamma_{1}, \mathbb{R}^{n}\right)$.

Definition 2 (cf. [13]). A subset $H_{0}$ of $\mathcal{L}^{0}\left(\bar{\Omega}, \mathbb{R}^{m}\right)_{\mu}$ is said to be $P C U$ stable if for any continuous partition of unity $\left(\alpha_{0}, \ldots, \alpha_{d}\right)$ with $\alpha_{0}, \ldots, \alpha_{d} \in$ $C^{\infty}(\bar{\Omega}, \mathbb{R})$, and any $\mathbf{z}_{0}, \ldots, \mathbf{z}_{d} \in H_{0}$, the sum $\sum_{i=0}^{d} \alpha_{i} \mathbf{z}_{i}$ is in $H_{0}$.

Proposition 3. The functional $\left(\widetilde{\mathcal{S}}_{\lambda}^{j}\right)^{* *}$ defined by (4.5)-(4.7) satisfies

$$
\begin{aligned}
& \left(\widetilde{\mathcal{S}}_{\lambda}^{j}\right)^{* *}\left(\varepsilon(\mathbf{u})_{\mid \Omega},-\gamma_{B}^{I}(\mathbf{u}) d s \otimes_{s} \boldsymbol{\nu}, \boldsymbol{\mu} \otimes_{s} \boldsymbol{\nu}\right)=-\int_{\Gamma_{1}} \lambda \mathbf{g} \cdot \boldsymbol{\mu} \\
& \left.\quad+\int_{\Gamma_{0}} j_{\infty}\left(x,-\gamma_{B}^{I}(\mathbf{u}) \otimes_{s} \boldsymbol{\nu}\right)\right) d s+\int_{\Gamma_{s}}\left(h_{s}^{*}+I_{\mathcal{K}}\right)^{*}\left(x,-\gamma_{B}^{I}(\mathbf{u})_{s} \boldsymbol{\nu}\right) d s \\
& \quad+\int_{\Gamma_{1}} j_{\infty}\left(x, \frac{d\left(\left(\boldsymbol{\mu}-\gamma_{B}^{I}(\mathbf{u}) d s\right) \otimes_{s} \boldsymbol{\nu}\right)}{d\left|\left(\boldsymbol{\mu}-\gamma_{B}^{I}(\mathbf{u}) d s\right) \otimes_{s} \boldsymbol{\nu}\right|}\right) d\left|\left(\boldsymbol{\mu}-\gamma_{B}^{I}(\mathbf{u}) d s\right) \otimes_{s} \boldsymbol{\nu}\right| \\
& \quad+\int_{\Omega} j\left(x, \varepsilon(\mathbf{u})_{a}\right) d x+\int_{\Omega} j_{\infty}\left(x, d \varepsilon(\mathbf{u})_{s} / d\left|\varepsilon(\mathbf{u})_{s}\right|\right) d\left|\varepsilon(\mathbf{u})_{s}\right|
\end{aligned}
$$


for every $\left(\varepsilon(\mathbf{u})_{\mid \Omega},-\gamma_{B}^{I}(\mathbf{u}) d s \otimes_{s} \boldsymbol{\nu}\right) \in \mathbf{Y}^{1}(\bar{\Omega})$ and $\boldsymbol{\mu} \otimes_{s} \boldsymbol{\nu} \in \mathbf{M}^{1}\left(\Gamma_{1}\right)$, where

$$
h_{s}^{*}(x, \boldsymbol{\sigma})= \begin{cases}0 & \text { if } \boldsymbol{\sigma}(x) \in \mathcal{A}(x), \\ \infty & \text { otherwise }\end{cases}
$$

and

$$
\begin{aligned}
\mathcal{A}(x)= & \left\{\boldsymbol{\sigma} \in \mathbf{E}_{s}^{n} \mid \sum_{i, j=1}^{n} \sigma_{i j}\left(\boldsymbol{\gamma}_{B}^{I}(\mathbf{u}) \otimes_{s} \boldsymbol{\nu}\right)_{i j} \geq 0 \text { for all } \mathbf{u}\right. \text { such that } \\
& \left.\sum_{i=1}^{n}\left(\boldsymbol{\gamma}_{B}^{I}(\mathbf{u}) \otimes_{s} \boldsymbol{\nu}\right)_{i i}=\gamma_{B}^{I}(\mathbf{u}) \cdot \boldsymbol{\nu} \leq 0\right\} \quad \text { for ds-a.e. } x \in \Gamma_{s} .
\end{aligned}
$$

Proof. Let $\mathbf{p} \equiv \boldsymbol{\mu}-\boldsymbol{\gamma}_{B}^{I}(\mathbf{u}) d s$ on $\Gamma_{1}$. By Assumption 6 we have $\boldsymbol{\beta}_{B}\left(\boldsymbol{\sigma}_{0}\right)=$ $\lambda \mathbf{g}$ on $\Gamma_{1}$. Hence $\int_{\Gamma_{1}} \lambda \mathbf{g} \cdot \boldsymbol{\mu}=\int_{\Gamma_{1}} \boldsymbol{\sigma}_{0}:\left(\boldsymbol{\mu} \otimes_{s} \boldsymbol{\nu}\right)$ for every $\boldsymbol{\mu} \in \mathbb{M}_{b}\left(\Gamma_{1}, \mathbb{R}^{n}\right)$. Note that $\gamma_{B}(L D(\Omega))=L^{1}\left(\operatorname{Fr} \Omega, \mathbb{R}^{n}\right)$ (cf. [23, Chapter 2, Theorem 1.1]). By [21, Theorem 3A], for every $\boldsymbol{\sigma} \in C_{\operatorname{div}}\left(\bar{\Omega}, \mathbf{E}_{s}^{n}\right)$ and $\boldsymbol{\kappa} \in C\left(\Gamma_{1}, \mathbf{E}_{s}^{n}\right)$ we get

$$
\begin{aligned}
& \quad\left(\widetilde{\mathcal{S}}_{\lambda}^{j}\right)^{*}(\boldsymbol{\sigma}, \boldsymbol{\kappa}) \equiv \sup \left\{\int_{\Gamma_{1}}(\boldsymbol{\kappa}-\boldsymbol{\sigma}):\left(\boldsymbol{\mu} \otimes_{s} \boldsymbol{\nu}\right)+\int_{\Gamma_{1}} \boldsymbol{\sigma}:\left(\mathbf{p} \otimes_{s} \boldsymbol{\nu}\right)\right. \\
& +\int_{\Gamma_{1}} \boldsymbol{\sigma}_{0}:\left(\boldsymbol{\mu} \otimes_{s} \boldsymbol{\nu}\right)-\int_{\Gamma_{1}} I_{\left\{\frac{d\left(\mathbf{p} \otimes_{s} \boldsymbol{\nu}\right)}{d\left|\mathbf{p} \otimes_{s} \boldsymbol{\nu}\right|}(x)=\mathbf{0}\right\}}\left(\frac{d\left(\mathbf{p} \otimes_{s} \boldsymbol{\nu}\right)}{d\left|\mathbf{p} \otimes_{s} \boldsymbol{\nu}\right|}\right) d\left|\mathbf{p} \otimes_{s} \boldsymbol{\nu}\right| \\
& +\int_{\Gamma_{0}} \boldsymbol{\sigma}:\left(-\boldsymbol{\gamma}_{B}^{I}(\mathbf{u}) \otimes_{s} \boldsymbol{\nu}\right) d s-\int_{\Gamma_{0}} I_{\left\{\boldsymbol{\gamma}_{B}^{I}(\mathbf{u})(x)=\mathbf{0}\right\}}\left(-\gamma_{B}^{I}(\mathbf{u}) \otimes_{s} \boldsymbol{\nu}\right) d s \\
& +\int_{\Gamma_{s}} \boldsymbol{\sigma}:\left(-\boldsymbol{\gamma}_{B}^{I}(\mathbf{u}) \otimes_{s} \boldsymbol{\nu}\right) d s-\int_{\Gamma_{s}} h_{s}\left(x,-\gamma_{B}^{I}(\mathbf{u}) \otimes_{s} \boldsymbol{\nu}\right) d s \\
& +\int_{\Omega} \boldsymbol{\sigma}: \mathbf{w} d x-\int_{\Omega} j(x, \mathbf{w}) d x \mid \mathbf{u} \in B D\left(\Omega_{1}\right) \text { such that } \mathbf{u}_{\mid \Omega} \in L D(\Omega), \\
& =\int_{\Omega} j^{*}(x, \boldsymbol{\sigma}) d x+\sup \left\{\int_{\Gamma_{1}}((\boldsymbol{\kappa}-\boldsymbol{\sigma}) \cdot \boldsymbol{\nu}) \cdot \boldsymbol{\mu}+\int_{\Omega_{1}}\left(\boldsymbol{\sigma}_{0} \cdot \boldsymbol{\nu}\right) \cdot \boldsymbol{\mu}\right. \\
& \left.+\int_{\Gamma_{1}} \boldsymbol{\sigma}:\left(\mathbf{p} \otimes_{s} \boldsymbol{\nu}\right) \mid \boldsymbol{\mu} \in \mathbb{M}_{b}\left(\Gamma_{1}, \mathbb{R}^{n}\right) \text { and } \mathbf{p} \otimes_{s} \boldsymbol{\nu}=\mathbf{0}\right\}+\int_{\Gamma_{s}} h_{s}^{*}(x, \boldsymbol{\sigma}) d s \\
& =\int_{\Omega} j^{*}(x, \boldsymbol{\sigma}) d x+\int_{\Gamma_{1}} I_{\{(\boldsymbol{\kappa}-\boldsymbol{\sigma}) \cdot \boldsymbol{\nu}(x)=-\lambda \mathbf{g}(x)\}}(\boldsymbol{\kappa}-\boldsymbol{\sigma}) d s+\int_{\Gamma_{s}} h_{s}^{*}(x, \boldsymbol{\sigma}) d s,
\end{aligned}
$$

because $\boldsymbol{\sigma}_{0} \cdot \boldsymbol{\nu}=\lambda \mathbf{g}$ on $\Gamma_{1}$ (see also [8, (4.9)] and [7, (3.18)]).

By the duality between $\mathbf{Y}^{1}(\bar{\Omega}) \times \mathbf{M}^{1}\left(\Gamma_{1}\right)$ and $C_{\operatorname{div}}\left(\bar{\Omega}, \mathbf{E}_{s}^{n}\right) \times C\left(\Gamma_{1}, \mathbf{E}_{s}^{n}\right)$ we obtain the functional $\left(\widetilde{\mathcal{S}}_{\lambda}^{j}\right)^{* *}$. The spaces $C_{\operatorname{div}}\left(\bar{\Omega}, \mathbf{E}_{s}^{n}\right)$ and $C\left(\Gamma_{1}, \mathbf{E}_{s}^{n}\right)$ are 
PCU-stable, so by [13, Theorem 1] and the proof of Theorem 4 of [13] we get

$$
\begin{aligned}
& \left(\widetilde{\mathcal{S}}_{\lambda}^{j}\right)^{* *}\left(\boldsymbol{\varepsilon}(\mathbf{u})_{\mid \bar{\Omega}}, \boldsymbol{\mu} \otimes_{s} \boldsymbol{\nu}\right)=\sup \left\{\int_{\Omega} \boldsymbol{\sigma}: \varepsilon(\mathbf{u})_{\mid \Omega}-\int_{\Omega} j^{*}(x, \boldsymbol{\sigma}) d x\right. \\
& +\int_{\Gamma_{1}}((\boldsymbol{\kappa}-\boldsymbol{\sigma}) \cdot \boldsymbol{\nu}) \cdot \boldsymbol{\mu}-\int_{\Gamma_{1}} I_{\{((\boldsymbol{\kappa}-\boldsymbol{\sigma}) \cdot \boldsymbol{\nu})(x)=-\lambda \mathbf{g}(x)\}}((\boldsymbol{\kappa}-\boldsymbol{\sigma}) \cdot \boldsymbol{\nu}) d s \\
& +\int_{\Gamma_{1}} \boldsymbol{\sigma}:\left[\left(\boldsymbol{\mu}-\gamma_{B}^{I}(\mathbf{u}) d s\right) \otimes_{s} \boldsymbol{\nu}\right]-\int_{\Gamma_{1}} j_{\infty}^{*}(x, \boldsymbol{\sigma}) d\left|\left(\boldsymbol{\mu}-\gamma_{B}^{I}(\mathbf{u}) d s\right) \otimes_{s} \boldsymbol{\nu}\right| \\
& +\int_{\Gamma_{0}} \boldsymbol{\sigma}:\left(-\gamma_{B}^{I}(\mathbf{u}) \otimes_{s} \boldsymbol{\nu}\right) d s-\int_{\Gamma_{0}} j_{\infty}^{*}(x, \boldsymbol{\sigma}) d s \\
& +\int_{\Gamma_{s}} \boldsymbol{\sigma}:\left(-\boldsymbol{\gamma}_{B}^{I}(\mathbf{u}) \otimes_{s} \boldsymbol{\nu}\right) d s-\int_{\Gamma_{s}}\left(h_{s}^{*}+j_{\infty}^{*}\right)(x, \boldsymbol{\sigma}) d s \mid \\
& \left.\boldsymbol{\sigma} \in C_{\operatorname{div}}\left(\bar{\Omega}, \mathbf{E}_{s}^{n}\right), \boldsymbol{\kappa} \in C\left(\Gamma_{1}, \mathbf{E}_{s}^{n}\right)\right\} \\
& =\sup \left\{\int_{\Omega}\left[\boldsymbol{\sigma}:\left(\varepsilon(\mathbf{u})_{a}\right)-j^{*}(x, \boldsymbol{\sigma})\right] d x\right. \\
& +\int_{\Omega}\left[\boldsymbol{\sigma}:\left(d \varepsilon(\mathbf{u})_{s} / d\left|\varepsilon(\mathbf{u})_{s}\right|\right)-j_{\infty}^{*}(x, \boldsymbol{\sigma})\right] d\left|\varepsilon(\mathbf{u})_{s}\right| \\
& -\int_{\Gamma_{1}} \lambda \mathbf{g} \cdot \boldsymbol{\mu}+\int_{\Gamma_{1}}\left[\boldsymbol{\sigma}:\left(d\left(\mathbf{p} \otimes_{s} \boldsymbol{\nu}\right) / d\left|\mathbf{p} \otimes_{s} \boldsymbol{\nu}\right|\right)-j_{\infty}^{*}(x, \boldsymbol{\sigma})\right] d\left|\mathbf{p} \otimes_{s} \boldsymbol{\nu}\right| \\
& +\int_{\Gamma_{s}}\left[\boldsymbol{\sigma}:\left(-\boldsymbol{\gamma}_{B}^{I}(\mathbf{u}) \otimes_{s} \boldsymbol{\nu}\right)-\left(h_{s}^{*}+j_{\infty}^{*}\right)(x, \boldsymbol{\sigma})\right] d s \\
& \left.+\int_{\Gamma_{0}}\left[\boldsymbol{\sigma}:\left(-\gamma_{B}^{I}(\mathbf{u}) \otimes_{s} \boldsymbol{\nu}\right)-j_{\infty}^{*}(x, \boldsymbol{\sigma})\right] d s \mid \boldsymbol{\sigma} \in C_{\operatorname{div}}\left(\bar{\Omega}, \mathbf{E}_{s}^{n}\right)\right\}
\end{aligned}
$$

for every $\boldsymbol{\varepsilon}(\mathbf{u})_{\mid \bar{\Omega}}=\left(\varepsilon(\mathbf{u})_{\mid \Omega},-\boldsymbol{\gamma}_{B}^{I}(\mathbf{u}) d s \otimes_{s} \boldsymbol{\nu}\right) \in \mathbf{Y}^{1}(\bar{\Omega})$ and $\left(\boldsymbol{\mu} \otimes_{s} \boldsymbol{\nu}\right) \in \mathbf{M}^{1}\left(\Gamma_{1}\right)$ (where $\mathbf{p} \equiv \boldsymbol{\mu}-\boldsymbol{\gamma}_{B}^{I}(\mathbf{u}) d s$ on $\Gamma_{1}$ ), which is (4.8). In the above calculations we use the equality $j_{\infty}^{*}(x, \boldsymbol{\sigma})=I_{\mathcal{K}(x)}(\boldsymbol{\sigma})=I_{\mathcal{K}}(x, \boldsymbol{\sigma})$, which holds for every $\boldsymbol{\sigma} \in \mathbf{E}_{s}^{n}$ and $x \in \bar{\Omega}$. Moreover, by (3.2) we can assume that $\boldsymbol{\sigma}(x) \in \mathcal{K}(x)$ for every $x \in \bar{\Omega}$. Thus the proof is complete.

Let $\mathbf{w} \in \mathbf{E}_{s}^{n}$. Then $w_{i j}^{D} \equiv w_{i j}-n^{-1} \delta_{i j} \operatorname{tr} \mathbf{w}$, where $\delta_{i j}$ is the Kronecker delta and $\mathbf{w}^{D}$ denotes the matrix $\left[w_{i j}^{D}\right]$.

Assumption 7 (cf. [10]). There exist functionals $j^{D}: \Omega \times\left(\mathbf{E}_{s}^{n}\right)^{D} \rightarrow$ $\mathbb{R} \cup\{\infty\}$ and $j^{\text {tr }}: \Omega \times \mathbb{R} \rightarrow \mathbb{R} \cup\{\infty\}$ such that there exist $k_{0}>0$ and $k_{1}>0$ such that for every $z \in \mathbb{R}$ and $d x$-a.e. $x \in \Omega, j^{\operatorname{tr}}(x, z) \geq k_{1} z^{2}-k_{0}$, and the normal integrand $j^{*}$ (given by Definition 1 and Assumption 3) satisfies 


$$
\left(j^{*}\right)^{*}(x, \boldsymbol{\varepsilon})=j(x, \boldsymbol{\varepsilon})=j^{D}\left(x, \boldsymbol{\varepsilon}^{D}\right)+j^{\operatorname{tr}}(x, \operatorname{tr} \boldsymbol{\varepsilon})
$$

for every $\varepsilon \in \mathbf{E}_{s}^{n}$ and $d x$-a.e. $x \in \Omega$.

We say that a net $\left\{\left(\mathbf{u}_{t}, \boldsymbol{\mu}_{t}\right)\right\}_{t \in T} \subset B D(\Omega) \times \mathbb{M}_{b}\left(\Gamma_{1}, \mathbb{R}^{n}\right)$ converges to $\left(\mathbf{u}_{0}, \boldsymbol{\mu}_{0}\right)$ in the weak ${ }^{*} B D(\Omega) \times \mathbb{M}_{b}\left(\Gamma_{1}, \mathbb{R}^{n}\right)$ topology if $\mathbf{u}_{t} \rightarrow \mathbf{u}_{0}$ in the weak ${ }^{*} B D(\Omega)$ topology and $\boldsymbol{\mu}_{t} \rightarrow \boldsymbol{\mu}_{0}$ in the weak ${ }^{*} \mathbb{M}_{b}\left(\Gamma_{1}, \mathbb{R}^{n}\right)$ topology.

Now we prove that $\left(\widetilde{\mathcal{S}}_{\lambda}^{j}\right)^{* *}$ is the l.s.c. regularization of $\mathcal{S}_{\lambda}^{j}$ in the weak* $B D(\Omega) \times \mathbb{M}_{b}\left(\Gamma_{1}, \mathbb{R}^{n}\right)$ topology, for the elastic-perfectly plastic potential $j$ given by (4.13). Recall that the space $U(\Omega)$ is given by

$$
U(\Omega) \equiv\left\{\mathbf{u} \in B D(\Omega) \mid \operatorname{div} \mathbf{u} \in L^{2}(\Omega)\right\} .
$$

Proposition 4 (cf. [10]). The functionals $\mathcal{S}_{\lambda}^{j}, \widetilde{\mathcal{S}}_{\lambda}^{j}$ and $\left(\widetilde{\mathcal{S}}_{\lambda}^{j}\right)^{* *}$ are equal to $\infty$ for every $\left(\varepsilon(\mathbf{u})_{\mid \Omega},-\gamma_{B}^{I}(\mathbf{u}) d s \otimes_{s} \boldsymbol{\nu}\right) \in \mathbf{Y}^{1}(\bar{\Omega})$ and $\boldsymbol{\mu} \otimes_{s} \boldsymbol{\nu} \in \mathbf{M}^{1}\left(\Gamma_{1}\right)$ such that $\mathbf{u}_{\mid \Omega} \in B D(\Omega)-U(\Omega)$.

Proof. By (4.13) we get

$$
\begin{aligned}
j^{*}\left(x, \mathbf{w}^{*}\right)= & \sup \left\{\mathbf{w}: \mathbf{w}^{*}-j^{D}\left(x, \mathbf{w}^{D}\right)-j^{\operatorname{tr}}(x, \operatorname{tr} \mathbf{w}) \mid \mathbf{w} \in \mathbf{E}_{s}^{n}\right\} \\
= & \sup \left\{\mathbf{w}^{D}:\left(\mathbf{w}^{*}\right)^{D}+n^{-1}(\operatorname{tr} \mathbf{w})\left(\operatorname{tr} \mathbf{w}^{*}\right)\right. \\
& \left.-j^{D}\left(x, \mathbf{w}^{D}\right)-j^{\operatorname{tr}}(x, \operatorname{tr} \mathbf{w}) \mid \mathbf{w} \in \mathbf{E}_{s}^{n}\right\} \\
= & \sup \left\{\mathbf{w}^{D}:\left(\mathbf{w}^{*}\right)^{D}-j^{D}\left(x, \mathbf{w}^{D}\right) \mid \mathbf{w} \in \mathbf{E}_{s}^{n}\right\} \\
& +\sup \left\{z\left(\operatorname{tr} \mathbf{w}^{*}\right) n^{-1}-j^{\operatorname{tr}}(x, z) \mid z \in \mathbb{R}^{\operatorname{tr}}\right\} \\
= & \left(j^{D}\right)^{*}\left(x,\left(\mathbf{w}^{*}\right)^{D}\right)+\left(j^{\operatorname{tr}}\right)^{*}\left(x, \operatorname{tr}\left(\mathbf{w}^{*}\right) n^{-1}\right) .
\end{aligned}
$$

Then by Assumption 7 there exist $k_{2}>0$ and $k_{3}>0$ such that for every $z \in \mathbb{R}$ and for $d x$-a.e. $x \in \Omega,\left(j^{\operatorname{tr}}\right)^{*}(x, z) \leq k_{2} z^{2}+k_{3}$. Thus by (3.2) there exists a multifunction $\mathcal{K}^{D}$ such that

$$
\mathcal{K}(x)=\mathcal{K}^{D}(x) \oplus\left\{z \mathbf{I} \in \mathbf{E}_{s}^{n} \mid I_{i j}=\delta_{i j} \text { and } z \in \mathbb{R}\right\},
$$

where $\mathcal{K}^{D}: \bar{\Omega} \rightarrow 2^{\left(\mathbf{E}_{s}^{n}\right)^{D}}$ is defined for every $x \in \bar{\Omega}$ (see (3.1)). By (3.5), we have $j_{\infty}(x, \mathbf{w})=\infty$ for every $x \in \bar{\Omega}$ and $\mathbf{w} \in \mathbf{E}_{s}^{n}$ such that $\operatorname{tr} \mathbf{w} \neq 0$. By (3.17), (4.5) and (4.8) we get $\mathcal{S}_{\lambda}^{j}\left(\varepsilon(\mathbf{u})_{\mid \bar{\Omega}}, \boldsymbol{\mu} \otimes_{s} \boldsymbol{\nu}\right)=\widetilde{\mathcal{S}}_{\lambda}^{j}\left(\varepsilon(\mathbf{u})_{\mid \Omega},-\gamma_{B}^{I}(\mathbf{u}) d s\right.$ $\left.\otimes_{s} \boldsymbol{\nu}, \boldsymbol{\mu} \otimes_{s} \boldsymbol{\nu}\right)=\left(\widetilde{\mathcal{S}}_{\lambda}^{j}\right)^{* *}\left(\varepsilon(\mathbf{u})_{\mid \Omega},-\gamma_{B}^{I}(\mathbf{u}) d s \otimes_{s} \boldsymbol{\nu}, \boldsymbol{\mu} \otimes_{s} \boldsymbol{\nu}\right)=\infty$ for every $\left(\varepsilon(\mathbf{u})_{\mid \bar{\Omega}}, \boldsymbol{\mu} \otimes_{s} \boldsymbol{\nu}\right)=\left(\varepsilon(\mathbf{u})_{\mid \Omega},-\gamma_{B}^{I}(\mathbf{u}) d s \otimes_{s} \boldsymbol{\nu}, \mu \otimes_{s} \boldsymbol{\nu}\right) \in \mathbf{Y}^{1}(\bar{\Omega}) \times \mathbf{M}^{1}\left(\Gamma_{1}\right)$ such that $\operatorname{tr} \varepsilon\left(\mathbf{u}_{\mid \Omega}\right)=\operatorname{div}\left(\mathbf{u}_{\mid \Omega}\right) \in \mathbb{M}_{b}(\Omega, \mathbb{R})-L^{1}(\Omega, \mathbb{R})$.

From (3.1), (3.5) and (4.16) we obtain

Corollary 5. For every $\mathbf{u} \in U(\Omega)$ and for ds-a.e. $x \in \operatorname{Fr} \Omega$,

$$
j_{\infty}\left(x,\left(-\gamma_{B}(\mathbf{u}) \otimes_{s} \boldsymbol{\nu}\right)\right)=\infty \quad \text { if }-\boldsymbol{\gamma}_{B}(\mathbf{u}) \cdot \boldsymbol{\nu} \neq 0
$$

on $\Gamma_{0}$, and 


$$
\begin{aligned}
\left(h_{s}^{*}+I_{\mathcal{K}}\right)^{*}\left(x,-\boldsymbol{\gamma}_{B}(\mathbf{u}) \otimes_{s} \boldsymbol{\nu}\right) & =h_{s}^{* *}\left(x,-\boldsymbol{\gamma}_{B}(\mathbf{u}) \otimes_{s} \boldsymbol{\nu}\right) \\
& =h_{s}\left(x,-\gamma_{B}(\mathbf{u}) \otimes_{s} \boldsymbol{\nu}\right)
\end{aligned}
$$

on $\Gamma_{s}$ (cf. Remark 2).

Now we turn to the direct method of regularization. The study of the difference between $\left(\mathcal{S}_{\lambda}^{j}\right)^{*}$ and $\left(\widetilde{\mathcal{S}}_{\lambda}^{j}\right)^{*}$ plays an essential role in this section.

Lemma 6. For every $\mathbf{u} \in B D\left(\Omega_{1}\right)$ and $\boldsymbol{\mu} \in \mathbb{M}_{b}\left(\Gamma_{1}, \mathbb{R}^{n}\right)$ such that $\mathbf{u}_{\mid \Omega} \in$ $L D(\Omega), \mathbf{u}_{\mid \Omega_{1}-\bar{\Omega}}=\mathbf{0}, \gamma_{B}^{I}(\mathbf{u})=\mathbf{0}$ on $\Gamma_{0}$ and $\boldsymbol{\gamma}_{B}^{I}(\mathbf{u}) d s=\boldsymbol{\mu}$ on $\Gamma$ we have

$$
\begin{aligned}
&\left(\widetilde{\mathcal{S}}_{\lambda}^{j}\right)^{* *}\left(\varepsilon(\mathbf{u})_{\mid \Omega},-\gamma_{B}^{I}(\mathbf{u}) d s \otimes_{s} \boldsymbol{\nu}, \boldsymbol{\mu} \otimes_{s} \boldsymbol{\nu}\right) \\
& \quad=\left(\mathcal{S}_{\lambda}^{j}\right)^{* *}\left(\varepsilon(\mathbf{u})_{\mid \bar{\Omega}}, \boldsymbol{\mu} \otimes_{s} \boldsymbol{\nu}\right)=\mathcal{S}_{\lambda}^{j}\left(\varepsilon(\mathbf{u})_{\mid \bar{\Omega}}, \boldsymbol{\mu} \otimes_{s} \boldsymbol{\nu}\right) .
\end{aligned}
$$

Proof. By Proposition 3 and by (4.18), we obtain (4.19) as in [8, Lemma 8] (see also [9, Lemma 1]).

Lemma 7. Let $\boldsymbol{\kappa}_{s} \in C\left(\Gamma_{1}, \mathbf{E}_{s}^{n}\right), \boldsymbol{\sigma}_{s} \in C_{\operatorname{div}}\left(\bar{\Omega}, \mathbf{E}_{s}^{n}\right)$, where $\operatorname{div} \boldsymbol{\sigma}_{s}=\mathbf{0}$ on $\Omega$ and $\int_{\Gamma_{1}} \boldsymbol{\kappa}_{s}:\left[\mathbf{t} \otimes_{s} \boldsymbol{\nu}\right] d s=0$ for every $\mathbf{t} \in L^{1}\left(\Gamma_{1}, \mathbb{R}^{n}\right)$. Then

$$
\left(\mathcal{S}_{\lambda}^{j}\right)^{*}(\boldsymbol{\sigma}, \boldsymbol{\kappa})=\left(\mathcal{S}_{\lambda}^{j}\right)^{*}\left(\boldsymbol{\sigma}+\boldsymbol{\sigma}_{s}, \boldsymbol{\kappa}+\boldsymbol{\kappa}_{s}\right)
$$

for every $\boldsymbol{\sigma} \in C_{\operatorname{div}}\left(\bar{\Omega}, \mathbf{E}_{s}^{n}\right)$ and $\boldsymbol{\kappa} \in C\left(\Gamma_{1}, \mathbf{E}_{s}^{n}\right)$.

Proof. By (4.2) and the Green formula (2.11) we have

$$
\begin{array}{r}
\left(\mathcal{S}_{\lambda}^{j}\right)^{*}(\boldsymbol{\sigma}, \boldsymbol{\kappa}) \\
=\sup \left\{-\int_{\Omega}(\operatorname{div} \boldsymbol{\sigma}) \cdot \mathbf{u} d x+\int_{\Gamma_{1}} \boldsymbol{\kappa}:\left(\boldsymbol{\mu} \otimes_{s} \boldsymbol{\nu}\right)-\mathcal{S}_{\lambda}^{j}\left(\varepsilon(\mathbf{u})_{\mid \bar{\Omega}}, \boldsymbol{\mu} \otimes_{s} \boldsymbol{\nu}\right) \mid\right. \\
\left.\mathbf{u}_{\mid \Omega} \in L D(\Omega), \mathbf{u}_{\mid \Omega_{1}-\bar{\Omega}}=\mathbf{0} \text { and } \boldsymbol{\mu} \in \mathbb{M}_{b}\left(\Gamma_{1}, \mathbb{R}^{n}\right)\right\} \\
=\sup \left\{-\int_{\Omega}\left[\operatorname{div}\left(\boldsymbol{\sigma}+\boldsymbol{\sigma}_{s}\right)\right] \cdot \mathbf{u} d x+\int_{\Gamma_{1}}\left(\boldsymbol{\kappa}+\boldsymbol{\kappa}_{s}\right):\left(\boldsymbol{\gamma}_{B}(\mathbf{u}) \otimes_{s} \boldsymbol{\nu}\right) d s\right. \\
-\mathcal{S}_{\lambda}^{j}\left(\varepsilon(\mathbf{u})_{\mid \bar{\Omega}}, \boldsymbol{\gamma}_{B}(\mathbf{u}) d s \otimes_{s} \boldsymbol{\nu}\right) \mid \\
\left.\mathbf{u}_{\mid \Omega} \in L D(\Omega), \mathbf{u}_{\mid \Omega_{1}-\bar{\Omega}}=\mathbf{0}\right\}=\left(\mathcal{S}_{\lambda}^{j}\right)^{*}\left(\boldsymbol{\sigma}+\boldsymbol{\sigma}_{s}, \boldsymbol{\kappa}+\boldsymbol{\kappa}_{s}\right)
\end{array}
$$

(cf. [7, Lemma 18] and (3.17)). Moreover, the trace $\gamma_{B}$ is a surjection from $L D(\Omega)$ onto $L^{1}\left(\operatorname{Fr} \Omega, \mathbb{R}^{n}\right)$ (see Theorem 1.1 of $[23$, Chapter 2$]$ ).

We say that a net $\left\{\left(\boldsymbol{\sigma}_{t}, \boldsymbol{\kappa}_{t}\right)\right\}_{t \in T} \subset C_{\operatorname{div}}\left(\bar{\Omega}, \mathbf{E}_{s}^{n}\right) \times C\left(\Gamma_{1}, \mathbf{E}_{s}^{n}\right)$ converges to $(\widehat{\boldsymbol{\sigma}}, \widehat{\boldsymbol{\kappa}})$ in the topology

$$
\begin{aligned}
& \sigma\left(C_{\operatorname{div}}\left(\bar{\Omega}, \mathbf{E}_{s}^{n}\right) \times C\left(\Gamma_{1}, \mathbf{E}_{s}^{n}\right), L^{1}\left(\Omega, \mathbf{E}_{s}^{n}\right)\right) \\
\times & \left.\left\{\left(\boldsymbol{\varphi}, \boldsymbol{\mu} \otimes_{s} \boldsymbol{\nu}\right) \in \mathbf{Y}^{1}(\bar{\Omega})_{\mid \operatorname{Fr} \Omega} \times \mathbf{M}^{1}\left(\Gamma_{1}\right) \mid \boldsymbol{\varphi}_{\mid \Gamma_{0}}=\mathbf{0}, \boldsymbol{\varphi}_{\mid \Gamma_{1}} d s=\boldsymbol{\mu} \otimes_{s} \boldsymbol{\nu}\right\}\right)
\end{aligned}
$$


if $\left\langle\left(\mathbf{w} d x, \widetilde{\mathbf{p}} \otimes_{s} \boldsymbol{\nu}, \widetilde{\mathbf{p}} d s \otimes_{s} \boldsymbol{\nu}\right) ;\left(\left(\boldsymbol{\sigma}_{t}, \boldsymbol{\kappa}_{t}\right)-(\widehat{\boldsymbol{\sigma}}, \widehat{\boldsymbol{\kappa}})\right)\right\rangle_{3} \rightarrow 0$ for all $\mathbf{w} \in L^{1}\left(\Omega, \mathbf{E}_{s}^{n}\right)$ and $\widetilde{\mathbf{p}} \in L^{1}\left(\Gamma_{1}, \mathbb{R}^{n}\right)$.

Lemma 8 (see [9, Lemma 3]). Let

$$
\widetilde{f}: C_{\text {div }}\left(\bar{\Omega}, \mathbf{E}_{s}^{n}\right) \times C\left(\Gamma_{1}, \mathbf{E}_{s}^{n}\right) \rightarrow \mathbb{R}
$$

be a linear functional, continuous in the topology (4.22), such that for some $\boldsymbol{\kappa}_{0} \in C\left(\Gamma_{1}, \mathbf{E}_{s}^{n}\right)$ and $\widetilde{\boldsymbol{\sigma}}_{1} \in C_{\operatorname{div}}\left(\bar{\Omega}, \mathbf{E}_{s}^{n}\right)$, and every $\boldsymbol{\sigma}_{s} \in C_{\operatorname{div}}\left(\bar{\Omega}, \mathbf{E}_{s}^{n}\right)$ with $\operatorname{div} \boldsymbol{\sigma}_{s}=\mathbf{0}\left(\right.$ in $\left.L^{n}\left(\Omega, \mathbb{R}^{n}\right)\right)$, we have

$$
\widetilde{f}\left(\widetilde{\boldsymbol{\sigma}}_{1}+\boldsymbol{\sigma}_{s}, \boldsymbol{\kappa}_{0}\right)=\widetilde{f}\left(\widetilde{\boldsymbol{\sigma}}_{1}, \boldsymbol{\kappa}_{0}\right) .
$$

Moreover, assume that for every $\boldsymbol{\sigma} \in C_{\operatorname{div}}\left(\bar{\Omega}, \mathbf{E}_{s}^{n}\right)$ and $\boldsymbol{\kappa}, \boldsymbol{\kappa}_{s} \in C\left(\Gamma_{1}, \mathbf{E}_{s}^{n}\right)$ such that $\int_{\Gamma_{1}} \boldsymbol{\kappa}_{s}:\left[\mathbf{t} \otimes_{s} \boldsymbol{\nu}\right] d s=0$ for every $\mathbf{t} \in L^{1}\left(\Gamma_{1}, \mathbb{R}^{n}\right)$, we have $\widetilde{f}(\boldsymbol{\sigma}, \boldsymbol{\kappa})=$ $\widetilde{f}\left(\boldsymbol{\sigma}, \boldsymbol{\kappa}+\boldsymbol{\kappa}_{s}\right)$. Then there exists $\widetilde{\mathbf{u}}_{1} \in L D(\Omega)$ such that $\boldsymbol{\gamma}_{B}\left(\widetilde{\mathbf{u}}_{1}\right)=\mathbf{0}$ on $\Gamma_{0}$ and for every $(\boldsymbol{\sigma}, \boldsymbol{\kappa}) \in C_{\mathrm{div}}\left(\bar{\Omega}, \mathbf{E}_{s}^{n}\right) \times C\left(\Gamma_{1}, \mathbf{E}_{s}^{n}\right)$ we have

$$
\begin{aligned}
\widetilde{f}(\boldsymbol{\sigma}, \boldsymbol{\kappa})= & \int_{\Omega} \boldsymbol{\sigma}: \boldsymbol{\varepsilon}\left(\widetilde{\mathbf{u}}_{1}\right) d x-\int_{\operatorname{Fr} \Omega} \boldsymbol{\sigma}:\left(\boldsymbol{\gamma}_{B}\left(\widetilde{\mathbf{u}}_{1}\right) \otimes_{s} \boldsymbol{\nu}\right) d s \\
& +\int_{\Gamma_{1}} \boldsymbol{\kappa}:\left(\boldsymbol{\gamma}_{B}\left(\widetilde{\mathbf{u}}_{1}\right) \otimes_{s} \boldsymbol{\nu}\right) d s .
\end{aligned}
$$

Let $Q_{1}: C_{\operatorname{div}}\left(\bar{\Omega}, \mathbf{E}_{s}^{n}\right) \times C\left(\Gamma_{1}, \mathbf{E}_{s}^{n}\right) \rightarrow \mathbb{R} \cup\{\infty\}$ be defined by

$$
Q_{1}(\boldsymbol{\sigma}, \boldsymbol{\kappa})=\inf _{\boldsymbol{\sigma}_{s}}\left\{\left(\widetilde{\mathcal{S}}_{\lambda}^{j}\right)^{*}\left(\boldsymbol{\sigma}+\boldsymbol{\sigma}_{s}, \boldsymbol{\kappa}\right) \mid \boldsymbol{\sigma}_{s} \in C\left(\bar{\Omega}, \mathbf{E}_{s}^{n}\right) \text { with } \operatorname{div} \boldsymbol{\sigma}_{s}=\mathbf{0}\right\}
$$
for $\boldsymbol{\sigma} \in C_{\mathrm{div}}\left(\bar{\Omega}, \mathbf{E}_{s}^{n}\right)$ and $\boldsymbol{\kappa} \in C\left(\Gamma_{1}, \mathbf{E}_{s}^{n}\right)$.

Proposition 9 (see $\left[9\right.$, Proposition 4]). For every $\boldsymbol{\sigma} \in C_{\operatorname{div}}\left(\bar{\Omega}, \mathbf{E}_{s}^{n}\right)$ and $\boldsymbol{\kappa} \in C\left(\Gamma_{1}, \mathbf{E}_{s}^{n}\right)$ we have

$$
\left(\mathcal{S}_{\lambda}^{j}\right)^{*}(\boldsymbol{\sigma}, \boldsymbol{\kappa})=\operatorname{cl}_{(4.22)} Q_{1}(\boldsymbol{\sigma}, \boldsymbol{\kappa})
$$

where $\operatorname{cl}_{(4.22)} Q_{1}$ denotes the largest minorant which is less than $Q_{1}$ and l.s.c. in the topology (4.22) (i.e. $\operatorname{cl}_{(4.22)} Q_{1}$ the l.s.c. regularization of $Q_{1}$ in (4.22)).

We say that a net $\left\{\left(\boldsymbol{\sigma}_{t}, \boldsymbol{\kappa}_{t}\right)\right\}_{t \in T} \subset C_{\mathrm{div}}\left(\bar{\Omega}, \mathbf{E}_{s}^{n}\right) \times C\left(\Gamma_{1}, \mathbf{E}_{s}^{n}\right)$ converges to $(\overline{\boldsymbol{\sigma}}, \overline{\boldsymbol{\kappa}})$ in the topology

$$
\sigma\left(C_{\operatorname{div}}\left(\bar{\Omega}, \mathbf{E}_{s}^{n}\right) \times C\left(\Gamma_{1}, \mathbf{E}_{s}^{n}\right), \mathbf{Y}^{1}(\bar{\Omega}) \times\left(L^{1}\left(\Gamma_{1}, \mathbb{R}^{n}\right) \otimes_{s} \boldsymbol{\nu}\right)\right.
$$

if

$$
\left\langle\left(\boldsymbol{\varepsilon}(\mathbf{u})_{\mid \bar{\Omega}}, \mathbf{z} d s \otimes_{s} \boldsymbol{\nu}\right) ;\left(\left(\boldsymbol{\sigma}_{t}, \boldsymbol{\kappa}_{t}\right)-(\overline{\boldsymbol{\sigma}}, \overline{\boldsymbol{\kappa}})\right)\right\rangle_{2} \rightarrow 0
$$

for every $\varepsilon(\mathbf{u})_{\mid \bar{\Omega}} \in \mathbf{Y}^{1}(\bar{\Omega})$ and $\mathbf{z} \in L^{1}\left(\Gamma_{1}, \mathbb{R}^{n}\right)$.

Proposition 10. Let $\bar{A}_{k} \equiv\left\{(\boldsymbol{\sigma}, \boldsymbol{\kappa}) \in C_{\operatorname{div}}\left(\bar{\Omega}, \mathbf{E}_{s}^{n}\right) \times C\left(\Gamma_{1}, \mathbf{E}_{s}^{n}\right) \mid\|\operatorname{div} \boldsymbol{\sigma}\|_{L^{n}}\right.$ $\left.\leq k,\|\boldsymbol{\kappa}\|_{C\left(\Gamma_{1}, \mathbf{E}_{s}^{n}\right)} \leq k\right\}$. For every $\widehat{\boldsymbol{\sigma}} \in C_{\operatorname{div}}\left(\bar{\Omega}, \mathbf{E}_{s}^{n}\right)$ and $\widehat{\boldsymbol{\kappa}} \in C\left(\Gamma_{1}, \mathbf{E}_{s}^{n}\right)$ there 
exists $k_{(\widehat{\boldsymbol{\sigma}}, \widehat{\boldsymbol{\kappa}})}>0$ such that for every $k \geq k_{(\widehat{\boldsymbol{\sigma}}, \widehat{\boldsymbol{\kappa}})}$,

$$
\left(\mathcal{S}_{\lambda}^{j}\right)^{*}(\widehat{\boldsymbol{\sigma}}, \widehat{\boldsymbol{\kappa}})=\operatorname{cl}_{\bar{A}_{k}} Q_{1}(\widehat{\boldsymbol{\sigma}}, \widehat{\boldsymbol{\kappa}}),
$$

where $\mathrm{cl}_{\bar{A}_{k}} Q_{1}(\cdot, \cdot)$ is the l.s.c. regularization of the function $(\boldsymbol{\sigma}, \boldsymbol{\kappa}) \mapsto$ $Q_{1}(\boldsymbol{\sigma}, \boldsymbol{\kappa})+I_{\bar{A}_{k}}(\boldsymbol{\sigma}, \boldsymbol{\kappa})$ in the topology $(4.22)$ and $I_{\bar{A}_{k}}(\cdot)$ is the indicator function of $\bar{A}_{k}$.

Proof. The proof is similar to that of [8, Proposition 13] (see also [9, Proposition 6]).

Theorem 11. For every $\varepsilon(\mathbf{u})_{\mid \bar{\Omega}} \in \mathbf{Y}^{1}(\bar{\Omega})$ and $\boldsymbol{\mu} \in \mathbb{M}_{b}\left(\Gamma_{1}, \mathbb{R}^{n}\right)$ we have

$$
\left(\widetilde{\mathcal{S}}_{\lambda}^{j}\right)^{* *}\left(\varepsilon(\mathbf{u})_{\mid \bar{\Omega}}, \boldsymbol{\mu} \otimes_{s} \boldsymbol{\nu}\right)=\left(\mathcal{S}_{\lambda}^{j}\right)^{* *}\left(\varepsilon(\mathbf{u})_{\mid \bar{\Omega}}, \boldsymbol{\mu} \otimes_{s} \boldsymbol{\nu}\right)
$$

Proof. The proof is similar to that of $[9$, Theorem 7] (see also [8, Theorem 14]).

The displacement formulation $\left(S N_{\lambda, j}\right)$ of the equilibrium problem for the elastic-plastic body made of a Hencky material with the Signorini contact condition is given by (3.7). The Suquet unilateral contact problem $\left(S S_{\lambda, j}\right)$ is defined by (3.10). Moreover, in [6] we studied the bidual relaxed Signorini problem

$$
\begin{array}{r}
\left(O P_{\lambda, j}^{* *}\right) \quad \text { Find } \inf \left\{\left(\lambda F_{s}\right)^{* *}(\mathbf{u}, \boldsymbol{\mu})+G_{j}^{* *}(\varepsilon(\mathbf{u})) \mid\right. \\
\left.\mathbf{u} \in B D(\Omega), \boldsymbol{\mu} \in \mathbb{M}_{b}\left(\Gamma_{1}, \mathbb{R}^{n}\right)\right\},
\end{array}
$$

where for every $\mathbf{u} \in B D(\Omega)$ and $\boldsymbol{\mu} \in \mathbb{M}_{b}\left(\Gamma_{1}, \mathbb{R}^{n}\right)$,

$$
\begin{aligned}
& \left(\lambda F_{s}\right)^{* *}(\mathbf{u}, \boldsymbol{\mu}) \equiv-\lambda\left[\int_{\Omega} \mathbf{f} \cdot \mathbf{u} d x+\int_{\Gamma_{1}} \mathbf{g} \cdot \boldsymbol{\mu}\right] \\
& \quad+\int_{\Gamma_{0}} j_{\infty}\left(x,-\gamma_{B}(\mathbf{u}) \otimes_{s} \boldsymbol{\nu}\right) d s+\int_{\Gamma_{s}}\left(h_{s}^{*}+I_{\mathcal{K}}\right)^{*}\left(x,-\gamma_{B}(\mathbf{u}) \otimes_{s} \boldsymbol{\nu}\right) d s \\
& \quad+\int_{\Gamma_{1}} j_{\infty}\left(x, \frac{d\left(\left(\boldsymbol{\mu}-\gamma_{B}(\mathbf{u}) d s\right) \otimes_{s} \boldsymbol{\nu}\right)}{d\left|\left(\boldsymbol{\mu}-\gamma_{B}(\mathbf{u}) d s\right) \otimes_{s} \boldsymbol{\nu}\right|}\right) d\left|\left(\boldsymbol{\mu}-\gamma_{B}(\mathbf{u}) d s\right) \otimes_{s} \boldsymbol{\nu}\right|
\end{aligned}
$$

and

$$
G_{j}^{* *}(\varepsilon(\mathbf{u}))=\int_{\Omega} j\left(x, \varepsilon(\mathbf{u})_{a}\right) d x+\int_{\Omega} j_{\infty}\left(x, \frac{d \varepsilon(\mathbf{u})_{s}}{d\left|\varepsilon(\mathbf{u})_{s}\right|}\right) d\left|\varepsilon(\mathbf{u})_{s}\right| .
$$

Lemma 12 (cf. Lemma 15 of [8]). If $\mathbf{f} \in L^{n+\delta}\left(\Omega, \mathbb{R}^{n}\right)$, where $\delta \geq 0$, then the functional $B D(\Omega) \ni \mathbf{u} \mapsto \int_{\Omega} \mathbf{f} \cdot \mathbf{u} d x \in \mathbb{R}$ is continuous in the weak $*^{*} B D(\Omega)$ and $\sigma\left(\mathbf{Y}^{1}(\bar{\Omega}), C_{\mathrm{div}}\left(\bar{\Omega}, \mathbf{E}_{s}^{n}\right)\right)$ topologies on bounded subsets of $B D(\Omega)$.

Assumption 8. There exist $k_{b}>0$ and $r_{1}>0$ such that $j^{*}\left(x, \mathbf{w}^{*}\right) \leq k_{b}$ for every $\mathbf{w}^{*} \in B_{\mathbf{E}_{s}^{n}}\left(0, r_{1}\right)$ and $d x$-a.e. $x \in \Omega$, where $B_{\mathbf{E}_{s}^{n}}\left(0, r_{1}\right)$ is the closed ball in $\mathbf{E}_{s}^{n}$ with center 0 and radius $r_{1}$. 
Suppose the function

$$
(\mathbf{u}, \boldsymbol{\mu}) \mapsto\left\lceil S S_{\lambda, j}\right\rceil(\mathbf{u}, \boldsymbol{\mu}) \equiv \lambda F_{2}(\mathbf{u}, \boldsymbol{\mu})+G_{j}(\varepsilon(\mathbf{u}))
$$

is coercive over $B D(\Omega) \times \mathbb{M}_{b}\left(\Gamma_{1}, \mathbb{R}^{n}\right)$, where $F_{2}$ and $G_{j}$ are given by $(3.11)$ and (3.9). Moreover, let $0 \leq \lambda_{1}<\lambda$. Then $\left\lceil S S_{\lambda_{1}, j}\right\rceil$ is coercive on $B D(\Omega) \times$ $\mathbb{M}_{b}\left(\Gamma_{1}, \mathbb{R}^{n}\right)$. Similarly, if the function

$$
(\mathbf{u}, \boldsymbol{\mu}) \mapsto\left\lceil O P_{\lambda, j}^{* *}\right\rceil(\mathbf{u}, \boldsymbol{\mu})=\left(\lambda F_{s}\right)^{* *}(\mathbf{u}, \boldsymbol{\mu})+G_{j}^{* *}(\varepsilon(\mathbf{u}))
$$

is coercive and $0 \leq \lambda_{1}<\lambda$, then $\left\lceil O P_{\lambda_{1}, j}^{* *}\right\rceil$ is coercive over $B D(\Omega) \times$ $\mathbb{M}_{b}\left(\Gamma_{1}, \mathbb{R}^{n}\right)$. Moreover,

$$
\left\lceil S S_{\lambda, j}\right\rceil(\mathbf{u}, \boldsymbol{\mu}) \geq\left\lceil O P_{\lambda, j}^{* *}\right\rceil(\mathbf{u}, \boldsymbol{\mu})
$$

for every $(\mathbf{u}, \boldsymbol{\mu}) \in B D(\Omega) \times \mathbb{M}_{b}\left(\Gamma_{1}, \mathbb{R}^{n}\right)$.

We say that a net $\left\{\left(\mathbf{u}_{t}, \boldsymbol{\mu}_{t}\right)\right\}_{t \in T} \subset B D(\Omega) \times \mathbb{M}_{b}\left(\Gamma_{1}, \mathbb{R}^{n}\right)$ converges to $\left(\mathbf{u}_{0}, \boldsymbol{\mu}_{0}\right)$ in the weak ${ }^{*} B D(\Omega) \times \mathbb{M}_{b}\left(\Gamma_{1}, \mathbb{R}^{n}\right)$ topology if $\mathbf{u}_{t} \rightarrow \mathbf{u}_{0}$ in the weak $^{*} B D(\Omega)$ topology and $\boldsymbol{\mu}_{t} \rightarrow \boldsymbol{\mu}_{0}$ in the weak $\mathbb{M}_{b}\left(\Gamma_{1}, \mathbb{R}^{n}\right)$ topology.

THEOREM 13. Let $\mathbf{f} \in L^{n+\delta}\left(\Omega, \mathbb{R}^{n}\right)$, where $\delta \geq 0$. If the function $\left\lceil S S_{\lambda, j}\right\rceil$ is coercive over $B D(\Omega) \times \mathbb{M}_{b}\left(\Gamma_{1}, \mathbb{R}^{n}\right)$, then the l.s.c. regularization of $\left\lceil S S_{\lambda, j}\right\rceil$ (in the weak $B D(\Omega) \times \mathbb{M}_{b}\left(\Gamma_{1}, \mathbb{R}^{n}\right)$ topology) is $\left\lceil O P_{\lambda, j}^{* *}\right\rceil$.

Proof. The proof is similar to that of [8, Theorem 18] and [9, Theorem 8].

Thus for every solution $\left(\mathbf{u}_{0}, \boldsymbol{\mu}_{0}\right)$ of $\left(O P_{\lambda, j}^{* *}\right)$ there is a net $\left\{\left(\mathbf{u}_{m}, \boldsymbol{\mu}_{m}\right)\right\}_{m \in \mathbf{H}}$ which minimizes $\left\lceil S S_{\lambda, j}\right\rceil$ and $\left(\mathbf{u}_{m}, \boldsymbol{\mu}_{m}\right) \rightarrow\left(\mathbf{u}_{0}, \boldsymbol{\mu}_{0}\right)$ in the weak ${ }^{*} B D(\Omega) \times$ $\mathbb{M}_{b}\left(\Gamma_{1}, \mathbb{R}^{n}\right)$ topology.

COROLlary 14. The functional $\left\lceil S S_{\lambda, j}\right\rceil$ is coercive over the space $B D(\Omega)$ $\times \mathbb{M}_{b}\left(\Gamma_{1}, \mathbb{R}^{n}\right)$ if and only if $\left\lceil O P_{\lambda, j}^{* *}\right\rceil$ is coercive.

Proof. By Theorem 13, if $\left\lceil S S_{\lambda, j}\right\rceil$ is coercive, then so is $\left\lceil O P_{\lambda, j}^{* *}\right\rceil$ (cf. Corollary 19 of [8]). By (4.37) the proof is complete.

The bidual relaxed Signorini problem (where the relaxation on $\Gamma_{1}$, proposed by Suquet in [22], is omitted) is defined by the formula

$$
\left(N P_{\lambda, j}^{* *}\right) \quad \text { Find } \inf \left\{\left(\lambda F_{N}\right)^{* *}(\mathbf{u})+G_{j}^{* *}(\varepsilon(\mathbf{u})) \mid \mathbf{u} \in B D(\Omega)\right\},
$$

where for every $\mathbf{u} \in B D(\Omega)$,

$$
\begin{aligned}
& \left(\lambda F_{N}\right)^{* *}(\mathbf{u}) \equiv-\lambda\left[\int_{\Omega} \mathbf{f} \cdot \mathbf{u} d x+\int_{\Gamma_{1}} \mathbf{g} \cdot \gamma_{B}(\mathbf{u}) d s\right] \\
& +\int_{\Gamma_{0}} j_{\infty}\left(x,-\gamma_{B}(\mathbf{u}) \otimes_{s} \boldsymbol{\nu}\right) d s+\int_{\Gamma_{s}}\left(h_{s}^{*}+I_{\mathcal{K}}\right)^{*}\left(x,-\gamma_{B}(\mathbf{u}) \otimes_{s} \boldsymbol{\nu}\right) d s
\end{aligned}
$$

and $G_{j}^{* *}$ is defined by (4.34).

Similarly to Theorem 13 (cf. [9, Theorem 18]) we obtain the following result: 
TheOREM 15. Let $\mathbf{f} \in L^{n+\delta}\left(\Omega, \mathbb{R}^{n}\right)$, where $\delta \geq 0$. If the function

$$
B D(\Omega) \ni \mathbf{u} \mapsto\left\lceil S N_{\lambda, j}\right\rceil(\mathbf{u})=\lambda F_{1}(\mathbf{u})+G_{j}(\varepsilon(\mathbf{u}))
$$

is coercive over $B D(\Omega)$, then the l.s.c. regularization of $\left\lceil S N_{\lambda, j}\right\rceil$ in the weak* $B D(\Omega)$ topology is the functional

$$
B D(\Omega) \ni \mathbf{u} \mapsto\left\lceil N P_{\lambda, j}^{* *}\right\rceil(\mathbf{u})=\left(\lambda F_{N}\right)^{* *}(\mathbf{u})+G_{j}^{* *}(\varepsilon(\mathbf{u})) .
$$

REMARK 1 . Let $\mathbf{f} \in L^{n+\delta}\left(\Omega, \mathbb{R}^{n}\right)$, where $\delta \geq 0$, and suppose the functional $\left\lceil S N_{\lambda, j}\right\rceil$ is coercive over $L D(\Omega)$. Since $\left\lceil S N_{\lambda, j}\right\rceil(\mathbf{u})=\infty$ for $\mathbf{u} \in$ $B D(\Omega)-L D(\Omega)$, the l.s.c. regularization of $\left\lceil S N_{\lambda, j}\right\rceil$ in the weak ${ }^{*} B D(\Omega)$ topology (over the space $S B D(\Omega)$ ) is

$$
S B D(\Omega) \ni \mathbf{u} \mapsto\left\lceil N P_{\lambda, j}^{* *}\right\rceil(\mathbf{u})=\left(\lambda F_{N}\right)^{* *}(\mathbf{u})+G_{j}^{* *}(\varepsilon(\mathbf{u}))
$$

(cf. [5]). Indeed, (4.42) is the supremum over the set of affine mappings (continuous in the weak ${ }^{*} B D(\Omega)$ topology) less than the functional $\left\lceil S N_{\lambda, j}\right\rceil$.

COROLlary 16. The functional $\left\lceil S N_{\lambda, j}\right\rceil$ is coercive over $B D(\Omega)$ if and only if $\left\lceil N P_{\lambda, j}^{* *}\right\rceil$ is coercive.

Proof. The proof is similar to that of Corollary 14.

COROLlary 17. The functional $\left\lceil S N_{\lambda, j}\right\rceil$ is coercive over $B D(\Omega)$ if and only if $\left\lceil O P_{\lambda, j}^{* *}\right\rceil$ is coercive over $B D(\Omega) \times \mathbb{M}_{b}\left(\Gamma_{1}, \mathbb{R}^{n}\right)$.

Proof. Indeed, by (3.7)-(3.11) the function $\left\lceil S S_{\lambda, j}\right\rceil$ is coercive over $B D(\Omega) \times \mathbb{M}_{b}\left(\Gamma_{1}, \mathbb{R}^{n}\right)$ if and only if $\left\lceil S N_{\lambda, j}\right\rceil$ is coercive over $B D(\Omega)$. By Corollary 14 the proof is complete.

ThEOREM 18. Let $\mathbf{f} \in L^{n+\delta}\left(\Omega, \mathbb{R}^{n}\right)$, where $\delta \geq 0$. Moreover, let $j^{*}: \Omega \times$ $\mathbf{E}_{s}^{n} \rightarrow \mathbb{R} \cup\{\infty\}$ be a nonnegative normal integrand. If the function $\left\lceil S N_{\lambda, j}\right\rceil$ is coercive over $B D(\Omega)$, then the couple $(\widetilde{\mathbf{u}}, \widetilde{\boldsymbol{\mu}}) \in B D(\Omega) \times \mathbb{M}_{b}\left(\Gamma_{1}, \mathbb{R}^{n}\right)$ is a solution of $\left(O P_{\lambda, j}^{* *}\right)$ if and only if $\widetilde{\boldsymbol{\mu}}=\gamma_{B}(\widetilde{\mathbf{u}})_{\mid \Gamma_{1}} d s \in \mathbb{M}_{b}\left(\Gamma_{1}, \mathbb{R}^{n}\right)$ and $\widetilde{\mathbf{u}}$ is a minimum point of the function $\left\lceil N P_{\lambda, j}^{* *}\right\rceil$.

Proof. Since $j^{*}$ is nonnegative, $\left(N P_{\lambda, j}^{* *}\right)$ and $\left(O P_{\lambda, j}^{* *}\right)$ have finite infima. Indeed, $\left\lceil N P_{\lambda, j}^{* *}\right\rceil(\mathbf{0})<\infty$ and $\left\lceil O P_{\lambda, j}^{* *}\right\rceil(\mathbf{0}, \mathbf{0})<\infty$ (cf. Assumptions 3 and 6$)$.

First, let $\widetilde{\mathbf{u}}$ be a solution of $\left(N P_{\lambda, j}^{* *}\right)$. Then the couple $\left(\widetilde{\mathbf{u}}, \gamma_{B}(\widetilde{\mathbf{u}})_{\mid \Gamma_{1}} d s\right) \in$ $B D(\Omega) \times \mathbb{M}_{b}\left(\Gamma_{1}, \mathbb{R}^{n}\right)$ is a solution of $\left(O P_{\lambda, j}^{* *}\right)$, since

$$
\inf \left(N P_{\lambda, j}^{* *}\right)=\inf \left(S N_{\lambda, j}\right)=\inf \left(S S_{\lambda, j}\right)=\inf \left(O P_{\lambda, j}^{* *}\right)
$$

(cf. Theorems 15 and 13 and formulae (3.7)-(3.11)).

Next, we show the converse implication. Let $(\widetilde{\mathbf{u}}, \widetilde{\boldsymbol{\mu}})$ be a solution of $\left(O P_{\lambda, j}^{* *}\right)$. Then there exists a sequence $\left\{\left(\mathbf{u}_{m}, \boldsymbol{\mu}_{m}\right)\right\}_{m \in \mathbb{N}}$ in $B D(\Omega) \times \mathbb{M}_{b}\left(\Gamma_{1}, \mathbb{R}^{n}\right)$ such that $\lim _{m \rightarrow \infty}\left\lceil S S_{\lambda, j}\right\rceil\left(\mathbf{u}_{m}, \boldsymbol{\mu}_{m}\right)=\inf \left(O P_{\lambda, j}^{* *}\right)$ and $\left(\mathbf{u}_{m}, \boldsymbol{\mu}_{m}\right) \rightarrow(\widetilde{\mathbf{u}}, \widetilde{\boldsymbol{\mu}})$ in the weak ${ }^{*} B D(\Omega) \times \mathbb{M}_{b}\left(\Gamma_{1}, \mathbb{R}^{n}\right)$ topology. By $(3.10)$ there exists $m_{0} \in \mathbb{N}$ such that for every $m>m_{0}$ we obtain $\boldsymbol{\mu}_{m}=\gamma_{B}\left(\mathbf{u}_{m}\right) d s$ on $\Gamma_{1}$, because 
$\inf \left(O P_{\lambda, j}^{* *}\right)<\infty$. Since $\inf \left(N P_{\lambda, j}^{* *}\right)=\inf \left(O P_{\lambda, j}^{* *}\right)$, we have

$$
\lim _{m \rightarrow \infty}\left\lceil S S_{\lambda, j}\right\rceil\left(\mathbf{u}_{m}, \gamma_{B}\left(\mathbf{u}_{m}\right)_{\mid \Gamma_{1}} d s\right)=\lim _{m \rightarrow \infty}\left\lceil S N_{\lambda, j}\right\rceil\left(\mathbf{u}_{m}\right)=\inf \left(N P_{\lambda, j}^{* *}\right)
$$

(cf. (4.43)). Therefore, by Theorem 15, we get

$$
\left\lceil N P_{\lambda, j}^{* *}\right\rceil(\widetilde{\mathbf{u}})=\inf \left(N P_{\lambda, j}^{* *}\right)=\inf \left(O P_{\lambda, j}^{* *}\right) .
$$

We now prove that $\widetilde{\boldsymbol{\mu}}=\boldsymbol{\gamma}_{B}(\widetilde{\mathbf{u}}) d s$ on $\Gamma_{1}$. Suppose otherwise. By (4.38), (4.32) and (4.45) we have $\left\lceil O P_{\lambda, j}^{* *}\right\rceil\left(\widetilde{\mathbf{u}}, \gamma_{B}(\widetilde{\mathbf{u}})_{\mid \Gamma_{1}} d s\right)=\left\lceil O P_{\lambda, j}^{* *}\right\rceil(\widetilde{\mathbf{u}}, \widetilde{\boldsymbol{\mu}})$. The function

$$
\begin{aligned}
{[0, \infty) \ni t \mapsto } & \int_{\Gamma_{1}} j_{\infty}\left(x, \frac{d\left(t\left(\widetilde{\boldsymbol{\mu}}-\gamma_{B}(\widetilde{\mathbf{u}}) d s\right) \otimes_{s} \boldsymbol{\nu}\right)}{d \mid\left(\widetilde{\boldsymbol{\mu}}-\gamma_{B}(\widetilde{\mathbf{u}}) d s\right) \otimes_{s} \boldsymbol{\nu}}\right) d\left|\left(\widetilde{\boldsymbol{\mu}}-\gamma_{B}(\widetilde{\mathbf{u}}) d s\right) \otimes_{s} \boldsymbol{\nu}\right| \\
& +(1-t) \int_{\Gamma_{1}} \mathbf{g} \cdot \gamma_{B}(\widetilde{\mathbf{u}}) d s+t \int_{\Gamma_{1}} \mathbf{g} \cdot \widetilde{\boldsymbol{\mu}}
\end{aligned}
$$

is positively homogeneous. Then

$$
\left\lceil O P_{\lambda, j}^{* *}\right\rceil(\widetilde{\mathbf{u}}, \tilde{\boldsymbol{\mu}})=\left\lceil O P_{\lambda, j}^{* *}\right\rceil\left(\widetilde{\mathbf{u}}, t\left(\widetilde{\boldsymbol{\mu}}-\gamma_{B}(\widetilde{\mathbf{u}})_{\mid \Gamma_{1}} d s\right)+\gamma_{B}(\widetilde{\mathbf{u}})_{\mid \Gamma_{1}} d s\right)
$$

for every $t>0$. This contradicts with the coercivity of $\left\lceil O P_{\lambda, j}^{* *}\right\rceil$ (cf. Corollary 17).

REMARK 2. In the case of soil mechanics, the set of admissible stresses is a convex cone which contains the half-line $A_{L} \equiv\left\{\boldsymbol{\sigma} \in \mathbf{E}_{s}^{n} \mid \operatorname{tr} \boldsymbol{\sigma} \leq 0\right.$, $\left.\boldsymbol{\sigma}^{D}=\mathbf{0}\right\}$, where $\boldsymbol{\sigma}^{D}=\boldsymbol{\sigma}-n^{-1} \boldsymbol{\delta} \operatorname{tr} \boldsymbol{\sigma}$ and $\delta_{i j}$ is the Kronecker delta. Moreover, by Assumption 8, the set of admissible stresses contains a neighborhood of $\mathbf{0} \in \mathbf{E}_{s}^{n}$ (the origin of the cone $\neq \mathbf{0}$ ). Then by direct calculation we obtain

$$
\begin{aligned}
\left(h_{s}^{*}+I_{\mathcal{K}_{1}}\right)^{*}\left(x,-\gamma_{B}(\mathbf{u}) \otimes_{s} \boldsymbol{\nu}\right) & =h_{s}^{* *}\left(x,-\gamma_{B}(\mathbf{u}) \otimes_{s} \boldsymbol{\nu}\right) \\
& =h_{s}\left(x,-\gamma_{B}(\mathbf{u}) \otimes_{s} \boldsymbol{\nu}\right),
\end{aligned}
$$

where $\mathcal{K}_{1}$ is the elastic domain (of the soil) at the point $x$. Indeed,

$$
\begin{aligned}
\sum_{i, j=1}^{n} \sigma_{i j}\left(\boldsymbol{\gamma}_{B}^{I}(\mathbf{u})\right. & \left.\otimes_{s} \boldsymbol{\nu}\right)_{i j} \\
= & \frac{1}{n} \operatorname{tr} \boldsymbol{\sigma} \operatorname{tr}\left(\boldsymbol{\gamma}_{B}^{I}(\mathbf{u}) \otimes_{s} \boldsymbol{\nu}\right)+\sum_{i, j=1}^{n} \sigma_{i j}^{D}\left(\boldsymbol{\gamma}_{B}^{I}(\mathbf{u}) \otimes_{s} \boldsymbol{\nu}\right)_{i j}^{D}
\end{aligned}
$$

and $\sup \left\{\boldsymbol{\sigma}:\left(-\boldsymbol{\gamma}_{B}^{I}(\mathbf{u})(x) \otimes_{s} \boldsymbol{\nu}\right)-I_{A_{L}}(\boldsymbol{\sigma}) \mid \boldsymbol{\sigma} \in \mathbf{E}_{s}^{n}\right\}=h_{s}\left(x,-\boldsymbol{\gamma}_{B}^{I}(\mathbf{u}) \otimes_{s} \boldsymbol{\nu}\right)$ (cf. (4.15)). Thus we obtain a generalization of Lemma 6 to the case of a soil material.

Let $\mathbf{f} \in L^{n+\delta}\left(\Omega, \mathbb{R}^{n}\right)$, where $\delta \geq 0$. Moreover, suppose the functions $\left\lceil S N_{\lambda, j}\right\rceil,\left\lceil N P_{\lambda, j}^{* *}\right\rceil,\left\lceil S S_{\lambda, j}\right\rceil$ and $\left\lceil O P_{\lambda, j}^{* *}\right\rceil$ describe a piece of elastic-plastic soil, i.e. $j, j_{\infty}, \mathcal{K}\left(\mathcal{K}=\mathcal{K}_{1}\right)$ describe the properties of the soil and Assumption 7 does not hold. If the function $\left\lceil S S_{\lambda, j}\right\rceil$ is coercive over $B D(\Omega) \times \mathbb{M}_{b}\left(\Gamma_{1}, \mathbb{R}^{n}\right)$, then its l.s.c. regularization in the weak ${ }^{*} B D(\Omega) \times \mathbb{M}_{b}\left(\Gamma_{1}, \mathbb{R}^{n}\right)$ topology 
is $\left\lceil O P_{\lambda, j}^{* *}\right\rceil$. Moreover, Theorems 13, 15, 18, Corollaries 14, 16 and 17 hold for the case of a soil material.

Suppose $d s\left(\Gamma_{0}\right)=0$ and $\int_{\Omega} \mathbf{f} \cdot \widehat{\mathbf{u}} d x+\int_{\Gamma_{1}} \mathbf{g} \cdot \gamma_{B}(\widehat{\mathbf{u}}) d s=0$ for every $\widehat{\mathbf{u}} \in \mathcal{R}_{0}$. Moreover, suppose that for every sequence $\left\{\left(\mathbf{u}_{m}, \boldsymbol{\mu}_{m}\right)\right\}_{m \in \mathbb{N}} \subset B D(\Omega) \times$ $\mathbb{M}_{b}\left(\Gamma_{1}, \mathbb{R}^{n}\right)$ such that $\inf _{\mathbf{r}}\left\{\left\|\mathbf{u}_{m}+\mathbf{r}\right\|_{B D}+\left\|\boldsymbol{\mu}_{m}+\gamma_{B}(\mathbf{r})_{\mid \Gamma_{1}} d s\right\|_{\mathbb{M}_{b}\left(\Gamma_{1}, \mathbb{R}^{n}\right)} \mid \mathbf{r} \in\right.$ $\left.\mathcal{R}_{0}\right\} \rightarrow \infty$ as $m \rightarrow \infty$, we have $\left\lceil O P_{\lambda, j}^{* *}\right\rceil\left(\mathbf{u}_{m}, \boldsymbol{\mu}_{m}\right) \rightarrow \infty$. Then there exists a solution $\left(\mathbf{u}_{s}, \boldsymbol{\mu}_{s}\right)$ of the problem $\left(O P_{\lambda, j}^{* *}\right)$ in $B D(\Omega) \times \mathbb{M}_{b}\left(\Gamma_{1}, \mathbb{R}^{n}\right)$. For every $\mathbf{u}^{1} \in \mathcal{R}_{0}$ the field $\left(\mathbf{u}_{s}+\mathbf{u}^{1}, \boldsymbol{\mu}_{s}+\gamma_{B}\left(\mathbf{u}^{1}\right)_{\mid \Gamma_{1}} d s\right) \in B D(\Omega) \times \mathbb{M}_{b}\left(\Gamma_{1}, \mathbb{R}^{n}\right)$ is a solution of $\left\lceil O P_{\lambda, j}^{* *}\right\rceil$ if and only if $\int_{\Gamma_{s}}\left(h_{s}^{*}+I_{\mathcal{K}}\right)^{*}\left(x,-\gamma_{B}\left(\mathbf{u}_{s}\right) \otimes_{s} \boldsymbol{\nu}\right) d s=$ $\int_{\Gamma_{s}}\left(h_{s}^{*}+I_{\mathcal{K}}\right)^{*}\left(x,-\gamma_{B}\left(\mathbf{u}_{s}+\mathbf{u}^{1}\right) \otimes_{s} \boldsymbol{\nu}\right) d s$ (cf. Theorems 4.34 and 4.31 of $\left.[6]\right)$.

\section{References}

[1] L. Ambrosio and G. Dal Maso, On the relaxation in $B V\left(\Omega, \mathbb{R}^{m}\right)$ of quasi-convex integrals, J. Funct. Anal. 109 (1992), 76-97.

[2] L. Ambrosio and D. Pallara, Integral representation of real functionals on $B V\left(\mathbb{R}^{n}, \mathbb{R}^{k}\right)$ and polyhedral approximation, Indiana Univ. Math. J. 42 (1993), 295-321.

[3] P. Aviles and Y. Giga, Variational integrals on mappings of bounded variation and their lower semicontinuity, Arch. Rat. Mech. Anal. 115 (1991), 201-255.

[4] C. Baiocchi, G. Buttazzo, F. Gastaldi and F. Tomarelli, General existence results for unilateral problems in continuum mechanics, ibid. 100 (1990), 149-189.

[5] A. C. Barroso, I. Fonseca and R. Toader, A relaxation theorem in the space of functions of bounded deformation, Ann. Scuola Norm. Sup. Pisa Cl. Sci. 29 (2000), 19-49.

[6] J. L. Bojarski, The relaxation of Signorini problems in Hencky plasticity, I: threedimensional solid, Nonlinear Anal. 29 (1997), 1091-1116.

[7] - General method of relaxation I: functionals defined on $B D(\Omega)$ space, Bull. Polish Acad. Sci. Math. 47 (1999), 289-301.

[8] —, General method of regularization. I: Functionals defined on BD space, Appl. Math. (Warsaw) 31 (2004), 175-199.

[9] —, General method of regularization. II: Relaxation proposed by Suquet, ibid., 321343.

[10] J. L. Bojarski and H. De Schepper, Discontinuities in Hencky plasticity: regularity of the solutions, Int. J. Appl. Math. 4 (2000), 27-37.

[11] G. Bouchitté, I. Fonseca and L. Mascarenhas, A global method for relaxation, Arch. Rat. Mech. Anal. 145 (1998), 51-98.

[12] - , - - - Relaxation of variational problems under trace constraints, Nonlinear Anal. 49 (2002), 221-246.

[13] G. Bouchitté and M. Valadier, Integral representation of convex functionals on a space of measures, J. Funct. Anal. 80 (1988), 398-420.

[14] N. Dunford and J. T. Schwartz, Linear Operators, Part I, Interscience, New York, 1958.

[15] G. Duvaut et J. L. Lions, Les Inéquations en Mécanique et en Physique, Dunod, Paris, 1972.

[16] I. Ekeland and R. Temam, Convex Analysis and Variational Problems, NorthHolland, Amsterdam and New York, 1976. 
[17] R. Engelking, General Topology, PWN-Polish Sci. Publ., Warszawa, 1977.

[18] I. Fonseca and S. Müller, Relaxation of quasiconvex functionals in $B V\left(\Omega, \mathbb{R}^{p}\right)$ for integrands $f(x, u, \nabla u)$, Arch. Rat. Mech. Anal. 123 (1993), 1-49.

[19] E. Giusti, Minimal Surfaces and Functions of Bounded Variation, lecture notes written by G. H. Williams, Dept. Math., Australian National Univ. 10, Canberra, 1977.

[20] R. Kohn and R. Temam, Dual spaces of stresses and strains with applications to Hencky plasticity, Appl. Math. Optim. 10 (1983), 1-35.

[21] R. T. Rockafellar, Integral functionals, normal integrands and measurable selections, in: Nonlinear Operators and the Calculus of Variations, Lecture Notes in Math. 543, Springer, Berlin, 1975, 157-207.

[22] P. M. Suquet, Discontinuities and plasticity, in: Non-Smooth Mechanics and Applications, J. J. Moreau and P. D. Panagiotopoulos (eds.), Springer, Wien, 1988, $278-340$.

[23] R. Temam, Mathematical Problems in Plasticity, Gauthier-Villars, Paris, 1985.

[24] R. Temam and G. Strang, Functions of bounded deformation, Arch. Rat. Mech. Anal. 75 (1980), 7-21.

[25] F. Tomarelli, Signorini problem in Hencky plasticity, Ann. Univ. Ferrara Sez. VII 36 (1990), 73-84.

Warsaw Agricultural University-SGGW

Department of Applied Mathematics

Nowoursynowska 159

02-787 Warszawa, Poland

E-mail: JarekLBojarski@poczta.onet.pl

Received on 21.10.2003;

revised version on 13.2.2004 